APS

physics

This is the accepted manuscript made available via CHORUS, the article has been published as:

Azimuthal distributions of charged hadrons, pions, and kaons produced in deep-inelastic scattering off unpolarized protons and deuterons

A. Airapetian et al. (HERMES Collaboration)

Phys. Rev. D 87, 012010 - Published 25 January 2013

DOI: 10.1103/PhysRevD.87.012010 


\title{
Azimuthal distributions of charged hadrons, pions, and kaons produced in deep-inelastic scattering off unpolarized protons and deuterons
}

A. Airapetian, ${ }^{13,16}$ N. Akopov, ${ }^{27}$ Z. Akopov, ${ }^{6}$ E.C. Aschenauer, ${ }^{7, *}$ W. Augustyniak, ${ }^{26}$ R. Avakian, ${ }^{27}$ A. Avetissian, ${ }^{27}$ E. Avetisyan, ${ }^{6}$ S. Belostotski, ${ }^{19}$ H.P. Blok,${ }^{18,}{ }^{25}$ A. Borissov ${ }^{6}$ J. Bowles, ${ }^{14}$ V. Bryzgalov,${ }^{20}$ J. Burns, ${ }^{14}$ M. Capiluppi, ${ }^{10}$ E. Cisbani, ${ }^{22}$ G. Ciullo, ${ }^{10}$ M. Contalbrigo,${ }^{10}$ P.F. Dalpiaz, ${ }^{10}$ W. Deconinck,${ }^{6}$ R. De Leo, ${ }^{2}$ L. De Nardo, ${ }^{12,6}$ E. De Sanctis,,${ }^{11}$ M. Diefenthaler,${ }^{15,}{ }^{9}$ P. Di Nezza, ${ }^{11}$ M. Düren, ${ }^{13}$ G. Elbakian, ${ }^{27}$ F. Ellinghaus ${ }^{5}$ A. Fantoni, ${ }^{11}$ L. Felawka, ${ }^{23}$ S. Frullani, ${ }^{22}$ G. Gapienko,${ }^{20}$ V. Gapienko, ${ }^{20}$ F. Garibaldi, ${ }^{22}$ G. Gavrilov $,{ }^{6}, 19,23$ V. Gharibyan, ${ }^{27}$ F. Giordano, ${ }^{15,10}$ S. Gliske, ${ }^{16}$ M. Golembiovskaya,${ }^{7}$ C. Hadjidakis, ${ }^{11}$ M. Hartig, ${ }^{6}$ D. Hasch, ${ }^{11}$ A. Hillenbrand, ${ }^{7}$ M. Hoek, ${ }^{14}$ Y. Holler, ${ }^{6}$ I. Hristova, ${ }^{7}$ Y. Imazu, ${ }^{24}$ A. Ivanilov, ${ }^{20}$ H.E. Jackson, ${ }^{1}$ H.S. Jo, ${ }^{12}$ S. Joosten, ${ }^{15,}{ }^{12}$ R. Kaiser $,{ }^{14}, \dagger$ G. Karyan, ${ }^{27}$ T. Keri, ${ }^{14},{ }^{13}$ E. Kinney, ${ }^{5}$ A. Kisselev, ${ }^{19}$ V. Korotkov ${ }^{20}$ V. Kozlov, ${ }^{17}$ P. Kravchenko, ${ }^{9,19}$ V.G. Krivokhijine, ${ }^{8}$ L. Lagamba, ${ }^{2}$ L. Lapikás, ${ }^{18}$ I. Lehmann, ${ }^{14}$ P. Lenisa, ${ }^{10}$ A. López Ruiz, ${ }^{12}$ W. Lorenzon, ${ }^{16}$ B.-Q. Ma,${ }^{3}$ D. Mahon, ${ }^{14}$ N.C.R. Makins, ${ }^{15}$ S.I. Manaenkov ${ }^{19}$ L. Manfré, ${ }^{22}$ Y. Mao,${ }^{3}$ B. Marianski, ${ }^{26}$ A. Martinez de la Ossa,${ }^{6,5}$ H. Marukyan,,${ }^{27}$ C.A. Miller,${ }^{23}$ Y. Miyachi, ${ }^{24, \ddagger}$ A. Movsisyan, ${ }^{27}$ M. Murray ${ }^{14}$ E. Nappi,${ }^{2}$ Y. Naryshkin, ${ }^{19}$ A. Nass,${ }^{9}$ M. Negodaev,${ }^{7}$ W.-D. Nowak,${ }^{7}$ L.L. Pappalardo, ${ }^{10}$ R. Perez-Benito, ${ }^{13}$ A. Petrosyan, ${ }^{27}$ M. Raithel,${ }^{9}$ P.E. Reimer, ${ }^{1}$ A.R. Reolon,,${ }^{11}$ C. Riedl,${ }^{7}$ K. Rith, ${ }^{9}$ G. Rosner, ${ }^{14}$ A. Rostomyan, ${ }^{6}$ J. Rubin,,${ }^{1,}{ }^{15}$ D. Ryckbosch, ${ }^{12}$ Y. Salomatin,${ }^{20}$ F. Sanftl,${ }^{24}$ A. Schäfer, ${ }^{21}$ G. Schnell, ${ }^{4,}{ }^{12}$ K.P. Schüler ${ }^{6}$ B. Seitz, ${ }^{14}$ T.-A. Shibata, ${ }^{24}$ M. Stancari, ${ }^{10}$ M. Statera,,${ }^{10}$ J.J.M. Steijger, ${ }^{18}$ J. Stewart, ${ }^{7}$ F. Stinzing, ${ }^{9}$ A. Terkulov ${ }^{17}$ R.M. Truty,${ }^{15}$ A. Trzcinski, ${ }^{26}$ M. Tytgat, ${ }^{12}$ A. Vandenbroucke, ${ }^{12}$ Y. Van Haarlem, ${ }^{12}$ C. Van Hulse, ${ }^{4}{ }^{12}$ D. Veretennikov, ${ }^{19}$ V. Vikhrov, ${ }^{19}$ I. Vilardi, ${ }^{2}$ S. Wang, ${ }^{3}$ S. Yaschenko, ${ }^{7,9}$ Z. Ye ${ }^{6}$ S. Yen, ${ }^{23}$ W. Yu, ${ }^{13}$ V. Zagrebelnyy, ${ }^{6,13}$ D. Zeiler, ${ }^{9}$ B. Zihlmann, ${ }^{6}$ and P. Zupranski ${ }^{26}$

(The Hermes Collaboration)

\author{
${ }^{1}$ Physics Division, Argonne National Laboratory, Argonne, Illinois 60439-4843, USA \\ ${ }^{2}$ Istituto Nazionale di Fisica Nucleare, Sezione di Bari, 70124 Bari, Italy \\ ${ }^{3}$ School of Physics, Peking University, Beijing 100871, China \\ ${ }^{4}$ Department of Theoretical Physics, University of the Basque Country UPV/EHU, 48080 \\ Bilbao, Spain and IKERBASQUE, Basque Foundation for Science, 48011 Bilbao, Spain \\ ${ }^{5}$ Nuclear Physics Laboratory, University of Colorado, Boulder, Colorado 80309-0390, USA \\ ${ }^{6}$ DESY, 22603 Hamburg, Germany \\ ${ }^{7}$ DESY, 15738 Zeuthen, Germany \\ ${ }^{8}$ Joint Institute for Nuclear Research, 141980 Dubna, Russia \\ ${ }^{9}$ Physikalisches Institut, Universität Erlangen-Nürnberg, 91058 Erlangen, Germany \\ ${ }^{10}$ Istituto Nazionale di Fisica Nucleare, Sezione di Ferrara and \\ Dipartimento di Fisica, Università di Ferrara, 44100 Ferrara, Italy \\ ${ }^{11}$ Istituto Nazionale di Fisica Nucleare, Laboratori Nazionali di Frascati, 00044 Frascati, Italy \\ ${ }^{12}$ Department of Physics and Astronomy, Ghent University, 9000 Gent, Belgium \\ ${ }^{13}$ Physikalisches Institut, Universität Gießen, 35392 Gießen, Germany \\ ${ }^{14}$ SUPA, School of Physics and Astronomy, University of Glasgow, Glasgow G12 8QQ, United Kingdom \\ ${ }^{15}$ Department of Physics, University of Illinois, Urbana, Illinois 61801-3080, USA \\ ${ }^{16}$ Randall Laboratory of Physics, University of Michigan, Ann Arbor, Michigan 48109-1040, USA \\ ${ }^{17}$ Lebedev Physical Institute, 117924 Moscow, Russia \\ ${ }^{18}$ National Institute for Subatomic Physics (Nikhef), 1009 DB Amsterdam, The Netherlands \\ ${ }^{19}$ B. P. Konstantinov Petersburg Nuclear Physics Institute, Gatchina, 188300 Leningrad Region, Russia \\ ${ }^{20}$ Institute for High Energy Physics, Protvino, 142281 Moscow Region, Russia \\ ${ }^{21}$ Institut für Theoretische Physik, Universität Regensburg, 93040 Regensburg, Germany \\ ${ }^{22}$ Istituto Nazionale di Fisica Nucleare, Sezione di Roma, Gruppo \\ Collegato Sanità and Istituto Superiore di Sanità, 00161 Roma, Italy \\ ${ }^{23}$ TRIUMF, Vancouver, British Columbia V6T 2A3, Canada \\ ${ }^{24}$ Department of Physics, Tokyo Institute of Technology, Tokyo 152, Japan \\ ${ }^{25}$ Department of Physics and Astronomy, VU University, $1081 \mathrm{HV}$ Amsterdam, The Netherlands \\ ${ }^{26}$ National Centre for Nuclear Research, 00-689 Warsaw, Poland \\ ${ }^{27}$ Yerevan Physics Institute, 375036 Yerevan, Armenia
}

\begin{abstract}
The azimuthal $\cos \phi$ and $\cos 2 \phi$ modulations of the distribution of hadrons produced in unpolarized semi-inclusive deep-inelastic scattering of electrons and positrons off hydrogen and deuterium targets have been measured in the HERMES experiment. For the first time these modulations were determined in a four-dimensional kinematic space for positively and negatively charged pions and kaons separately, as well as for unidentified hadrons. These azimuthal dependences are sensitive
\end{abstract}


to the transverse motion and polarization of the quarks within the nucleon via, e.g., the Cahn, Boer-Mulders and Collins effects.

PACS numbers: 13.88.+e, 13.60.-r

Keywords: Semi-inclusive deep-inelastic scattering, azimuthal modulations, intrinsic transverse momentum and spin.

\section{INTRODUCTION}

Since the late 1960s the quark-parton model [1, 2] has been used to describe the structure of the nucleon in terms of fundamental constituents. Their behavior inside the nucleon was parametrized in terms of parton distribution functions (PDFs). Historically PDFs depended only on the fractional quark momentum longitudinal to the nucleon direction of motion, $x$, and on the scale at which the distributions were probed, $Q^{2}$, while transverse degrees of freedom were neglected. These PDFs have provided a good description of processes in which transverse spin and momentum are integrated over [3-5]. However, transverse degrees of freedom are not a priori negligible and are needed for a complete description of the nucleon. To account for transverse motion the PDFs have been generalized to transverse-momentumdependent PDFs, known also as TMDs [6-11].

Already in the early days of the parton model it was realized that the inclusion of quark intrinsic transverse momentum, $p_{T}$, leads to modifications of the cross sections in high-energy reactions involving hadrons in the initial state, e.g., in lepton-nucleon deep-inelastic scattering (DIS) $[12,13]$. In particular, in semi-inclusive DIS transverse momenta give rise to azimuthal dependences of the distribution of the produced hadrons about the direction of the virtual photon $[6,8,13-16]$. In 1978, $\mathrm{R}$. Cahn discussed the emergence of cosine modulations in the semi-inclusive DIS cross section in the presence of non-vanishing transverse parton momentum using simple kinematic considerations (Cahn effect) [17, 18]. Once included, interplay between the parton transverse momentum and the partons's and nucleon's spins can generate further azimuthal asymmetries, as, e.g., in the BoerMulders effect. The Boer-Mulders mechanism was introduced for the first time in 1997 [8] in relation to naive-T-odd effects ${ }^{1}[19,20]$. For a long time naive-Todd effects were believed to vanish due to time-reversal invariance [21]. Recently, it was shown that final and initial-state interactions can produce naive-T-odd effects without violating T-invariance [22-24]. Because it involves only the parton spin and not the nucleon spin,

\footnotetext{
* Now at: Brookhaven National Laboratory, Upton, New York 11772-5000, USA

$\dagger$ Present address: International Atomic Energy Agency, $1400 \mathrm{Vi}-$ enna, Austria

¥ Now at: Department of Physics, Yamagata University Yamagata, 990-8560, Japan

1 A naive-T-odd transformation is defined to be T-odd in the usual sense except without the interchange of initial and final states.
}

the Boer-Mulders mechanism is a good example of how spin-related effects may play an important role, even in unpolarized reactions. Measurements of these novel correlations provide insights into the so far poorly explored partonic transverse degrees of freedom and can be used to gather information, in a model-dependent way, about the elusive parton orbital motion.

In DIS the structure of the nucleon is probed by the interaction of a high-energy lepton $(l)$ with a target nucleon $(N)$ via the exchange of electroweak bosons. In the kinematic region accessed at HERMEs it is a good approximation to consider only the exchange of a single photon (Born approximation) [25]. In semi-inclusive DIS measurements, at least one of the hadrons $(h)$ produced in the collision is detected in coincidence with the scattered lepton $\left(l^{\prime}\right)$ :

$$
l+N \rightarrow l^{\prime}+h+X,
$$

where $X$ represents the remaining, unobserved, final state. The polarization-averaged semi-inclusive DIS cross section can be written in a model-independent way by means of four structure functions [26, 27]:

$$
\begin{aligned}
& d \sigma_{U U} \equiv \frac{\mathrm{d}^{5} \sigma_{U U}}{\mathrm{~d} x \mathrm{~d} y \mathrm{~d} z \mathrm{~d} P_{h \perp}^{2} \mathrm{~d} \phi}= \\
& 2 \pi \frac{\alpha^{2}}{x y Q^{2}} \frac{y^{2}}{2(1-\epsilon)}\left(1+\frac{\gamma^{2}}{2 x}\right)\left\{F_{U U, T}+\epsilon F_{U U, L}\right. \\
& \left.+\sqrt{2 \epsilon(1+\epsilon)} F_{U U}^{\cos \phi} \cos \phi+\epsilon F_{U U}^{\cos 2 \phi} \cos 2 \phi\right\} .
\end{aligned}
$$

Here, $-Q^{2}$ is the squared four-momentum carried by the virtual photon. In the target rest frame, $y$ is the fraction of the beam energy carried by the virtual photon and $z$ is the fraction of the virtual photon energy carried by the produced hadron. The hadron momentum component transverse to the virtual photon direction is denoted $P_{h \perp}$, and $\phi$ is the azimuthal angle of the hadron production plane around the virtual photon direction with respect to the lepton scattering plane (see Fig. 1). The quantity $\alpha$ is the electromagnetic coupling constant, and $\gamma=2 M x / Q$ with $M$ the proton mass. The structure functions $F_{U U, T}, F_{U U, L}, F_{U U}^{\cos \phi}, F_{U U}^{\cos 2 \phi}$ depend on $x, Q^{2}$, $z$ and $P_{h \perp}$; the subscript $U U$ stands for unpolarized beam and target, while $T(L)$ indicates transverse (longitudinal) polarization of the virtual photon, and $\epsilon$ is the ratio of longitudinal to transverse photon flux.

Besides intrinsic transverse parton momentum, perturbative-QCD (pQCD) effects, like gluon radiation, will also lead to azimuthal dependences in the semi-inclusive DIS cross section [29, 30]. However, they contribute mainly at large values of $P_{h \perp}$, and are 


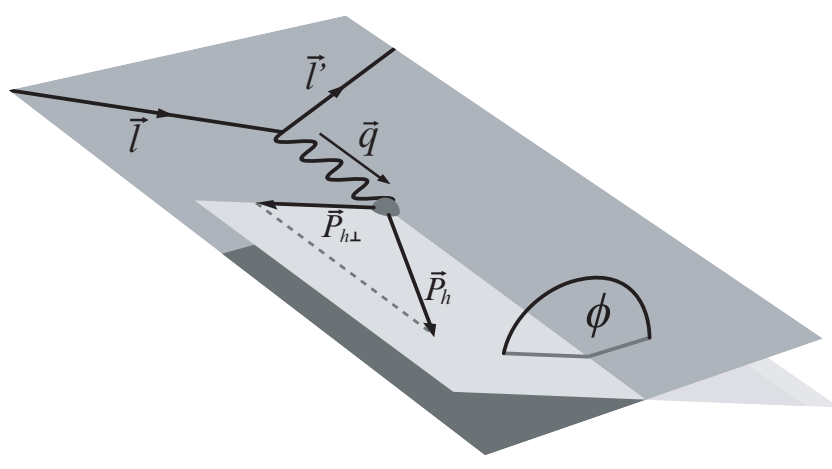

FIG. 1. Depiction of the azimuthal angle $\phi$ between the scattering plane, spanned by the three-momenta $\left(\vec{l}, \overrightarrow{l^{\prime}}\right)$ of incoming and outgoing leptons and the hadron plane, defined by the respective three-momenta of the virtual photon and the produced hadron, $\vec{q}$ and $\overrightarrow{P_{h}}$, defined according to the Trento convention [28].

next-to-leading order in the strong coupling constant. For hadron transverse momenta that are small compared to the hard scale $Q\left(P_{h \perp} \ll Q\right)$, TMD factorization $[7,9,10]$ allows for an expansion of the structure functions in powers of $1 / Q$ and expresses them in terms of convolutions of transverse-momentum dependent distribution and fragmentation functions.

The TMDs parametrize the nucleon structure and the fragmentation functions describe how the struck parton evolves into the observed hadronic final state. For simplicity, in the following the weak $Q^{2}$ dependence of the TMDs is not explicitly written. Each convolution can be classified according to the suppression, in powers of $1 / Q$, at which they contribute to the structure function. Not all contributions from all possible suppression levels have been calculated yet. In this work primarily contributions up to a suppression of $(1 / Q)$ will be considered, but, considering the low average $Q^{2}$ attainable at Hermes, contributions suppressed as $(1 / Q)^{2}$ or higher may be not negligible.

The structure function related to the $\cos 2 \phi$ amplitude, $F_{U U}^{\cos 2 \phi}$, receives a single unsuppressed contribution:

$$
F_{U U}^{\cos 2 \phi} \propto-\sum_{q}\left[h_{1}^{\perp, q}\left(x, p_{T}^{2}\right) \otimes \mathcal{W}_{1} H_{1}^{\perp, q}\left(z, k_{T}^{2}\right)\right] .
$$

Additional contributions are present only at a suppression of $\propto(1 / Q)^{2}$, or higher. The sum symbol, $\sum_{q}$, stands for a quark-charge-squared weighted sum over quark flavors. The symbol $\otimes \mathcal{W}_{1}$ represents a weighted ${ }^{2}$ convolution integral over the intrinsic momentum $p_{T}$ and over

\footnotetext{
${ }^{2}$ The weights $\mathcal{W}_{1}$ (Eq. 3), $\mathcal{W}_{2}$ (Eq. 4), and $\mathcal{W}_{3}$ and $\mathcal{W}_{4}$ (Eq. 5) are kinematic factors depending on $p_{T}$ and $k_{T}$; for their complete expressions see Ref. [27].
}

$k_{T}$, the momentum transverse to the struck quark direction that the hadron acquires during the fragmentation process.

The contribution shown in Eq. 3 is called the BoerMulders-Collins effect (also often referred to as simply the Boer-Mulders effect). It involves the BoerMulders distribution function $h_{1}^{\perp, q}\left(x, p_{T}^{2}\right)$ [8], which describes the correlation between the transverse polarization and transverse momentum of quarks in an unpolarized nucleon, and the Collins fragmentation function $H_{1}^{\perp, q}\left(z, k_{T}^{2}\right)$ [21], which describes the probability for a transversely polarized quark to fragment to an unpolarized hadron with a certain transverse-momentum direction; both these functions are chiral-odd. As hard QED and hard QCD interactions preserve chirality, two chiralodd functions need to appear in conjunction, in order to have a chiral-even observable.

As discussed above, naive-T-odd observables can be non-zero in conjunction with final- or initial-state interactions, which are reflected by the presence of non-trivial gauge links in the definition of the TMDs [23, 24]. This gauge link leads to the direct QCD prediction that naiveT-odd distribution functions must have opposite signs in semi-inclusive DIS and Drell-Yan reactions [23]. To date, this sign change has not yet been confirmed.

There are no contributions to $F_{U U}^{\cos 2 \phi}$ at a suppression $1 / Q$. Not all contributions beyond a suppression of $1 / Q$ have been calculated, however a term

$$
\propto\left(\frac{M}{Q}\right)^{2} \sum_{q}\left[f_{1}^{q}\left(x, p_{T}^{2}\right) \otimes_{\mathcal{W}_{2}} D_{1}^{q}\left(z, k_{T}^{2}\right)\right]
$$

arising from the Cahn effect $[17,18]$ is expected. The Cahn effect has recently received increasing attention, as it can provide information about the average transverse momentum of unpolarized quarks in unpolarized hadrons. It involves the convolution over transverse momenta of the spin-averaged distribution and fragmentation functions, $f_{1}^{q}\left(x, p_{T}^{2}\right)$ and $D_{1}^{q}\left(z, k_{T}^{2}\right)$, respectively. Their transverse-momentum-integrated correspondents, $f_{1}^{q}(x)$ and $D_{1}^{q}(z)$, are well known [31,32]. However, their $p_{T^{-}}$and $k_{T^{-}}$-dependences are poorly constrained by measurements, and thus the convolution integrals in Eq. 4 can be estimated only approximately. Moreover, the average intrinsic transverse momentum $\left\langle p_{T}\right\rangle$ may depend on the parton flavor; thus a flavor-dependent measure of the Cahn effect, via, e.g., semi-inclusive DIS of identified hadrons, is highly desirable.

The first non-zero contributions to the structure function $F_{U U}^{\cos \phi}$ in Eq. 2, which is related to a $\cos \phi$ amplitude, are suppressed as $1 / Q$; subsequent contributions are suppressed as $(1 / Q)^{3}$. Among the various contributions suppressed as $1 / Q$, several involve either a distribution or fragmentation function that relates to quarkgluon-quark correlations, and hence is interaction dependent and has no probabilistic interpretation. In the Wandzura-Wilczeck approximation [33] all these terms 
are neglected, and only two contributions are considered:

$$
\begin{aligned}
F_{U U}^{\cos \phi} \simeq & -\frac{M}{Q} \sum_{q}\left[h_{1}^{\perp, q}\left(x, p_{T}^{2}\right) \otimes_{\mathcal{W}_{3}} H_{1}^{\perp, q}\left(z, k_{T}^{2}\right)\right] \\
& -\frac{M}{Q} \sum_{q}\left[f_{1}^{q}\left(x, p_{T}^{2}\right) \otimes_{\mathcal{W}_{4}} D_{1}^{q}\left(z, k_{T}^{2}\right)\right] .
\end{aligned}
$$

In the first line of Eq. 5 the Boer-Mulders-Collins effect is recognizable, while, in the second line, the Cahn effect is present.

Only a few measurements of $\cos 2 \phi$ and $\cos \phi$ amplitudes in semi-inclusive DIS experiments have been published over the past 30 years [34-37]. Most measurements averaged over any possible flavor dependence as they refer to hadrons without type nor charge distinction, and only hydrogen target [34-36] or hydrogen and deuterium targets combined together [37] were available. Recently, the CLAS collaboration measured non-zero cosine modulations for positive pions [38] produced by semi-inclusive DIS off the proton. The Compass collaboration presented preliminary $\cos 2 \phi$ and $\cos \phi$ amplitudes in semiinclusive DIS [39] but has not yet published final results. In Drell-Yan experiments non-zero azimuthal modulations have been measured [40-45] that violate the LamTung relation [46]. Such a violation can be ascribed to the Boer-Mulders distribution function, as pointed out in Ref. [47]. Sizable modulations have been extracted in pion-induced Drell-Yan reactions, where a valence quark and a valence antiquark annihilate. When a sea parton is involved, as in proton-induced Drell-Yan processes, the measured modulations become smaller, suggesting a small Boer-Mulders function for the sea.

This paper presents cosine modulations for positively and negatively charged unidentified hadrons as well as for identified charged pions and kaons produced by DIS off hydrogen and deuterium targets.

\section{THE HERMES EXPERIMENT}

The cosine modulations described in the previous section were extracted from measurements performed at the fixed-target HERMES experiment. HERMES acquired data from 1995 to 2007 with various polarized and unpolarized gaseous targets internal to the HERA 27.6 GeV electron/positron storage ring at DESY. In this paper results are presented that were extracted using only the pure hydrogen and deuterium targets, where the lepton beam scatters directly off neutrons and protons (with only negligible nuclear effects in case of deuterium). The spectrometer [48] was a forward-angle instrument consisting of two symmetric halves above and below the horizontal plane defined by the lepton-beam pipe. Particles with polar angles within $\pm 170 \mathrm{mrad}$ in the horizontal direction and between $\pm(40-140)$ mrad vertically could be detected. The collected data were processed with a tracking code involving event-level fitting based on a Kalman-filter algorithm [49], which corrects the tracking parameters
TABLE I. Numbers of charged hadrons for each data set (in

\begin{tabular}{|c|c|c|c|c|c|}
\hline \multicolumn{6}{|c|}{ Data set } \\
\hline Year: & 2000 & 2005 & 2006 & 2007 & \multirow{2}{*}{ Total } \\
\hline Beam: & $\mathrm{e}^{+}$ & $\mathrm{e}^{-}$ & $\mathrm{e}^{+}$ & $\mathrm{e}^{+}$ & \\
\hline \multicolumn{6}{|c|}{ Hydrogen target } \\
\hline $\mathrm{h}^{+}$ & 0.80 & - & 1.97 & 2.11 & 4.88 \\
\hline $\mathrm{h}^{-}$ & 0.45 & - & 1.12 & 1.20 & 2.77 \\
\hline$\pi^{+}$ & 0.57 & - & 1.42 & 1.53 & 3.52 \\
\hline$\pi^{-}$ & 0.40 & - & 0.99 & 1.07 & 2.46 \\
\hline $\mathrm{K}^{+}$ & 0.10 & - & 0.24 & 0.26 & 0.60 \\
\hline $\mathrm{K}^{-}$ & 0.03 & - & 0.08 & 0.09 & 0.20 \\
\hline \multicolumn{6}{|c|}{ Deuterium target } \\
\hline $\mathrm{h}^{+}$ & 1.02 & 0.52 & 0.48 & 0.55 & 2.57 \\
\hline $\mathrm{h}^{-}$ & 0.66 & 0.34 & 0.31 & 0.36 & 1.67 \\
\hline$\pi^{+}$ & 0.72 & 0.38 & 0.35 & 0.40 & 1.85 \\
\hline$\pi^{-}$ & 0.58 & 0.31 & 0.28 & 0.32 & 1.49 \\
\hline $\mathrm{K}^{+}$ & 0.12 & 0.06 & 0.06 & 0.07 & 0.31 \\
\hline $\mathrm{K}^{-}$ & 0.04 & 0.02 & 0.02 & 0.03 & 0.11 \\
\hline
\end{tabular}
millions).

for the effects from magnetic fields and accounts for all detector materials and known mis-alignments.

Lepton-hadron separation with an efficiency better than $98 \%$ was achieved using the combination of several detectors: a transition-radiation detector [48], a dualradiator ring-imaging Cherenkov $(\mathrm{RICH})$ detector [50, 51], a lead and scintillator preshower detector, and a lead-glass calorimeter [52]. Hadron identification was performed using the RICH detector, taking into account the entire event topology simultaneously, rather than a single particle at a time. This provides improved particle identification compared to earlier algorithms (see appendix B for further details).

\section{DATA ANALYSIS}

\section{A. Data selection}

The data used for this work were collected during the 2000-2007 periods, with both lepton beam charges and unpolarized hydrogen and deuterium targets, as summarized in table I. In order to guarantee high-quality data, each event had to meet several criteria, such as good performance of the particle identification and tracking detectors. Each selected track satisfies geometric constraints to ensure that it originated from the beam-target interaction region and also remained well within the acceptance of the spectrometer.

Events with at least one lepton and one hadron detected in coincidence are included in the semi-inclusive 
DIS event sample if they satisfy the following kinematic requirements. The DIS region is defined here by the kinematic constraints $Q^{2}>1 \mathrm{GeV}^{2}$ and $W^{2}>10 \mathrm{GeV}^{2}$, where $W^{2}$ is the squared invariant mass of the initial system of virtual photon and target nucleon. As a consequence of these requirements and the limited angular acceptance of HERMES, $x$ and $y$ are restricted to the ranges $0.023<x<0.6$ and $0.2<y$. In addition the restriction $y<0.85$ is applied, dictated by the energy threshold in the calorimeter to ensure high trigger efficiency. In order to suppress hadrons not originating from the struck quark (i.e., to suppress those from the target fragmentation region), the requirements $z>0.2$ and $x_{F}>0.2$ are applied. Here, $x_{F}=2 p_{z} / \sqrt{s}$ is the Feynman scaling variable, where $p_{z}$ is the hadron momentum component parallel to the virtual photon, and $\sqrt{s}$ is the total energy in the $\gamma^{*} p$ center-of-mass system.

To ensure good identification of hadrons by the RICH, identified pions are required to have momenta within $1 \mathrm{GeV}<P_{h}<15 \mathrm{GeV}$ and kaons within $2 \mathrm{GeV}<P_{h}<15 \mathrm{GeV}$. RICH weights are assigned to each hadron. These weights correspond to the probabilities that the hadron is a pion or a kaon, as determined from the RICH hadron type hypothesis and the identification efficiency, computed from a Monte Carlo simulation of the RICH detector, which has been tuned to data. No RICH identification or RICH weights are applied for the data sample of unidentified hadrons. To be consistent with the pion sample, the momentum restriction $1 \mathrm{GeV}<P_{h}<15 \mathrm{GeV}$ is also applied to unidentified hadrons. In the calculation of all kinematic quantities that require particle masses, the pion mass was used for unidentified hadrons. This is as a good approximation as $70 \%$ (88\%) of positive (negative) hadrons are pions (see table I).

The selected event sample is corrected for contamination from leptons that do not originate from the scattered beam but rather come from lepton-pair production in detector material or meson Dalitz decay $\left(\pi^{0} / \eta \rightarrow \gamma e^{+} e^{-}\right)$. These events amount to less than $1 \%$ of the total number of events, and are typically concentrated toward the high$y$ region, where their contribution reaches $8 \%$. These contaminating processes are charge symmetric. Therefore events passing DIS selection but with the wrong lepton charge constitute a control sample which is kinematically matched to the background events wrongly included in the semi-inclusive DIS sample. The correction is performed by assigning a negative weight to the events with lepton charge opposite to that of the beam.

\section{B. Extraction procedure}

Experimentally, the azimuthal modulations of the unpolarized cross section can be accessed via the $\cos n \phi$ - moments $(n=1,2)$

$$
\langle\cos n \phi\rangle_{U U}=\frac{\int_{0}^{2 \pi} \cos n \phi d \sigma_{U U} \mathrm{~d} \phi}{\int_{0}^{2 \pi} d \sigma_{U U} \mathrm{~d} \phi},
$$

where $d \sigma_{U U}$ is defined in Eq. 2. The moments are related to the structure functions of interest via the $\phi$ independent part of the cross section

$$
\begin{aligned}
F_{U U}^{\cos \phi} & =\frac{2\langle\cos \phi\rangle}{\sqrt{2 \epsilon(1+\epsilon)}}\left(F_{U U, T}+\epsilon F_{U U, L}\right), \\
F_{U U}^{\cos 2 \phi} & =\frac{2\langle\cos 2 \phi\rangle}{\epsilon}\left(F_{U U, T}+\epsilon F_{U U, L}\right) .
\end{aligned}
$$

Extracting the cosine modulations of the unpolarized cross section from data requires disentangling them from a number of experimental sources of azimuthal modulations. At Hermes, due to the separation of the spectrometer into two symmetric top-bottom halves, the azimuthal acceptance is non-uniform. In addition, the observed kinematic conditions of each event may differ from the Born conditions at the hard electromagnetic vertex due to both physical and experimental effects. Events may be reconstructed with altered kinematic conditions due to external bremsstrahlung and multiple scattering in the detector material, or due to initial or final state radiation from the beam lepton (higher-order QED effects). All of these effects lead to a miscalculation of the kinematic conditions, and can induce false $\cos n \phi$ modulations.

To correct the data for kinematic smearing and false cosine modulations, a binned unfolding procedure was applied. A large Pythia6 [53] Monte Carlo simulation (with approximately 20 times more events than the experimental data) was generated, which uses the JETSET [54] fragmentation model tuned to Hermes kinematic conditions [55]. This simulation includes QED radiative effects calculated with RADGEN [56], and a complete Geant3 [57] simulation of the HeRmes spectrometer. All known instrumental and reconstruction effects are simulated, including particle interactions with detector materials and detector responses that account for known inefficiencies. The simulated events then pass through the HERMES event-reconstruction algorithm, mimicking any possible tracking bias or inefficiency present in the data. The simulated semi-inclusive DIS sample provides information on both the Born-level and the observed smeared kinematic conditions, and thus it can be used to build a matrix that describes the migration of events between kinematic bins. This simulation is also used to define the events that smear into the measurement from outside the accepted kinematic range, and thus represent background events in each kinematic bin.

A smearing matrix is constructed by normalizing the migration matrix to the Born cross section, taken from a Born-level Pythia6 production, in each bin. As a result of this normalization, the smearing matrix is a relative quantity, and reflects the fraction of events that are 
within the Hermes acceptance and their bin-by-bin migration. In the limit of infinitely small bins, the smearing matrix is independent of the cross section model used to build it. However, the kinematic distribution of the background events depends on the Born cross section model used to describe events generated outside the Hermes acceptance. The model dependence of the smearing matrix (due to finite sized bins) and the background are discussed in section IV B.

The simulated samples are normalized relative to the data via the inclusive DIS cross section, as determined by the LEPTO Monte Carlo generator [58]. To correct the data for events smeared into the acceptance, the background events are subtracted from the normalized yields. The smearing matrix is then used to unfold the background-subtracted data, correcting for QED radiative effects, detector effects, acceptance and all the detector inefficiencies included in the simulation.

The functional form

$$
\mathcal{A}+\mathcal{B} \cos \phi+\mathcal{C} \cos 2 \phi
$$

is fit to the azimuthal distribution of the unfolded yields to extract the cosine modulations $\langle\cos \phi\rangle_{U U}=\mathcal{B} / 2 \mathcal{A}$ and $\langle\cos 2 \phi\rangle_{U U}=\mathcal{C} / 2 \mathcal{A}$. As the unfolding procedure is a linear operation, it can be combined with the linear operation of fitting. This was done with linear regression, where a $\chi^{2}$ was formed:

$$
\chi^{2}=\left(\sigma^{\text {data }}-S X \beta\right)^{T} C^{-1}\left(\sigma^{\text {data }}-S X \beta\right) .
$$

Here, $\sigma^{\text {data }}$ is the measured, background-subtracted yield, $S$ is the smearing matrix, and $C$ is a covariance matrix that includes the statistical uncertainties of data and background, and the statistical precision of the Monte Carlo used to construct the smearing matrix. The product $X \beta$ gives the fit function of Eq. 9 representing the Born-level event yield, with $\beta$ the vector of parameters $(\mathcal{A}, \mathcal{B}$, and $\mathcal{C})$ and $X$ the block diagonal design matrix that includes a constant term (equal to 1 ) and the values of $\cos \langle\phi\rangle$ and $\cos 2\langle\phi\rangle$ for each $\phi$ bin. See appendix A 1 for a detailed explanation of these matrices.

The running conditions at HERMES changed from year to year. Thus the data of each year must be independently unfolded with the proper Monte Carlo production, including the appropriate experimental configuration. To combine the results from the various data sets, the formalism of Eq. 10 has been extended to a procedure that at the same time unfolds each data set independently and fits the Born-level yields from all years simultaneously. Technical details about the full procedure are provided in appendix A 1.

As the Born cross section depends on five kinematic variables (see Eq. 2), this procedure is carried out on a five dimensional grid of kinematic bins. An analysis in fewer dimensions would implicitly integrate over variables and mix together physics and experimental effects. This mixing can obscure the true signal, as demonstrated by Monte Carlo tests. A Monte Carlo simulation with
TABLE II. Kinematic bin boundaries

\begin{tabular}{rlllllll}
\hline \hline $\mathrm{x}:$ & 0.023 & 0.042 & 0.078 & 0.145 & 0.27 & 0.6 & \\
$\mathrm{y}:$ & 0.2 & 0.3 & 0.45 & 0.6 & 0.7 & 0.85 & \\
$\mathrm{z}:$ & 0.2 & 0.3 & 0.4 & 0.5 & 0.6 & 0.75 & 1.0 \\
$P_{h \perp}[\mathrm{GeV}]:$ & 0.05 & 0.2 & 0.35 & 0.5 & 0.7 & 1.0 & 1.3 \\
$\phi:$ & 12 equidistant bins & & & & \\
\hline \hline
\end{tabular}

an isotropic distribution in $\phi$ at the Born level was run through the detector simulation. When unfolded in less than five dimensions, false modulations were extracted, which were of similar size as the physical moments seen in the data [59].

The binning used is reported in table II. After the fit to the $\phi$-dependence, the final four-dimensional ( $4 D$ ) cosine modulations represent fully differential results. Due to the unfolding procedure the results in the various kinematic bins are statistically correlated as well as the results for the $\langle\cos \phi\rangle$ and $\langle\cos 2 \phi\rangle$ moments in each bin due to the fitting procedure. Therefore, the complete covariance matrix must be considered to avoid overestimating the statistical uncertainties in results projected on fewer dimensions.

\section{SYSTEMATIC UNCERTAINTIES}

This section discusses the systematic uncertainties related to the imperfect treatment of instrumental bias and inefficiencies by the smearing matrices. Systematic contributions related to residual model dependence of the unfolding procedure due to finite bin sizes are also evaluated.

In contrast to the case of unidentified hadrons, the systematic uncertainties for identified pions and kaons include an additional contribution from the RICH identification. In these cases, the Monte Carlo studies described below include a full simulation of the RICH detector.

\section{A. Instrumental effects}

The geometric acceptance of the HeRMES spectrometer produces cosine modulations larger than the measured signals; therefore a number of systematic checks have been performed and are listed in this section.

The experimental apparatus experienced several major changes over time. The lepton beam charge changed as HERA alternated between accelerating electrons and positrons. For the last two years of data taking, the target cell was shifted in the beam-line direction closer to the forward spectrometer, and its length, initially of $40 \mathrm{~cm}$, was reduced by a factor of two. Different magnetic fields were active in the target region in different years. The cosine modulations were extracted from data collected without any target magnetic field and with lon- 
gitudinal magnetic fields: a solenoid of 0.3 Tm strength, employed for a longitudinally polarized target in 2000, and the 1.0 Tm solenoid of the Hermes recoil detector installed after 2005. In addition, during shut-down periods some detectors were moved in and out, and relative positions between detectors changed. All these altered conditions induce changes in the geometric acceptance; therefore each data taking period requires a dedicated simulation to properly correct for the acceptance.

Despite those significant changes in running conditions, the cosine modulations extracted separately for each year are found to be mostly consistent. Small systematic shifts between years are observed, which can be ascribed to effects not included in the simulations, and thus in the correction. These effects include residual detector misalignment not accounted for in the tracking algorithm, which are expected to change from one datataking period to another.

The time stability of the apparatus response was checked by measuring the azimuthal modulations generated by the HERMES acceptance in short time intervals within the same data taking period. The test indicated that the azimuthal modulations of the acceptance are stable in time.

The tiny instabilities $(<1 \%$ of the observed amplitudes) are highly dependent on the year under study, and, as the Monte Carlo does not simulate or correct for any of these instabilities, they can partially explain the small differences between cosine modulations extracted from different years.

To take these differences into account, the signed difference between moments extracted from each year and moments extracted from a simultaneous fit of the remaining periods was evaluated at the $4 D$-level. The modulus of the weighted average of these differences was added to the systematic uncertainty, and represents the largest contribution to the systematic uncertainty ( $\sim 70 \%$ of the total systematic uncertainty).

The extracted moments were checked for a sensitivity to a possible beam misalignment or slope with respect to the spectrometer axis, and misplacement of the spectrometer dipole magnet. No significant effects have been found. The net beam polarization was found to be negligible. Additional instrumental sources that could generate false azimuthal modulations have been tested by measuring sine modulations and cosine modulations higher than $\cos 2 \phi$, which are not present in the unpolarized semi-inclusive DIS cross section in the single-photon exchange approximation. No significant signals were found.

The final moments discussed in section V and VI have not been corrected for possible binning effects or for RICH inefficiencies or cross-contaminations that were not accounted for (for identified pion and kaon samples only), for example due to $\phi$-dependence not accounted for in the RICH weights. The influence of these effects on the final moments was estimated by a Monte Carlo simulation. For each particle type under study, a $4 D$ model of the measured cosine modulations was extracted from the fully differential final moments by means of a $4 D$ parameterization (details in appendix A2). Through an accept/reject procedure, those models were used to alter, at the Born-level, the underlying distribution in an originally azimuthally uniform PYTHIA6 production that includes the full spectrometer simulation and QED radiative effects. For the identified hadron cases, both pion and kaon models were implemented, to account for cross-contaminations between modulations. The protons constitute the remaining significant part of the hadron sample, but a model was not extracted for them. The sensitivity of the test to proton modulations was checked by implementing a model for protons that was either $\phi$ independent or with modulations identical to that of pions. The proton model input to the Monte Carlo is found to have very little impact on the test results.

This simulation, modeled to reproduce the measured cosine azimuthal modulations, was used as a surrogate for the data in the entire analysis procedure, and cosine modulations for pions and kaons and unidentified hadrons were extracted. The extracted moments were found to agree with the input models, verifying that the unfolding algorithm is able to extract the implemented modulations after correcting for all instrumental and QED radiative effects included in the simulation. The small discrepancies between the extracted moments and the input model provide an estimate of systematic uncertainty due to the unfolding procedure, binning effects and RICH weights (in the case of the identified pion and kaon samples).

\section{B. Model dependence}

The unfolding procedure described in section III B can be affected by two different sources of model dependence. The unfolding correction is based mainly on two objects: a smearing matrix, describing the migration of events between bins, and a background estimation, describing the events that are smeared into the kinematic bins from outside the geometric/kinematic acceptance.

In a fully differential analysis and in the limit of infinitely narrow bins, the smearing matrix is independent of the models underlying the Monte Carlo event generator used to produce it. Residual model dependence due to finite bin sizes was tested by comparing data azimuthal moments extracted using smearing matrices computed with different models for the azimuthal dependent part of the cross section: the standard, $\phi$-independent, PYтHIA6 cross section, and the altered Pythia6 cross section that includes the $4 D$ cosine model extracted from data, as described in section IV A. As expected, no significant differences were observed in the extracted moments.

To test the model dependence of the background a similar procedure was used, and cosine modulations extracted with different models for background evaluation were compared. Again the two models used were the standard, $\phi$-independent Pyтнia6 cross section, and the Pythia6 cross section modified to include the $4 D$ cosine 
model, which was extrapolated into the unmeasured region not covered by the detector acceptance. The moments from data were found to be weakly sensitive to the azimuthal dependence of the background. The differences between the moments extracted with the two models were used to estimate the systematic uncertainties from the model dependence and were combined with the other systematic uncertainties in quadrature.

\section{Calculation of systematic uncertainties}

The systematic uncertainties described above are subject to statistical fluctuations due to the finite statistical precision of the Monte Carlo simulations used to calculate them. To average out these statistical fluctuations, each systematic contribution was smoothed by fitting it to a $4 D$ linear function. Higher order $4 D$ polynomials were tested, and provided final systematic uncertainties of similar size. The final systematic uncertainty was calculated by adding each smoothed contribution in quadrature.

\section{FULLY DIFFERENTIAL RESULTS}

The $4 D$ analysis described in section IIIB provides access to the full kinematic dependences. The final moments, in four dimensions, for positive and negative unidentified hadrons, pions, and kaons produced from hydrogen and deuterium targets, are available online [60]. These fully differential moments represent the complete set of results of this analysis and can be used to test theoretical models.

The moments are accompanied by the covariance matrix describing the statistical correlations, the total systematic uncertainties for each bin (all contributions are added in quadrature), and the average $\langle x\rangle,\langle y\rangle,\langle z\rangle$, $\left\langle P_{h \perp}\right\rangle$, and $\left\langle Q^{2}\right\rangle$ values for each bin. It is not possible to make a measurement in every $\left(x, y, z, P_{h \perp}\right)$ bin due to (a) kinematic constraints that exclude some portions of the $4 D$ space, (b) the not-uniform distribution of the underlying cross section across the rectangular kinematic binning, (c) the detector acceptance, and (d) the limited statistical precision of the data. Bins that do not contain enough events to make a measurement (and typically also have statistical uncertainties larger than unity) are denoted in the database with all moments and average kinematics set to zero. In the covariance matrix, elements corresponding to such bins have diagonal element values of one and non-diagonal element values of zero.

A visual representation of the bins where a measurement is not possible can be found online [61] for the statistically poorest data set for each particle type (pions, kaons, and all hadrons). This tool also allows the user to integrate the moments in an arbitrary kinematic range (following the procedure described in the next section).
TABLE III. Kinematic ranges of integration.

\begin{tabular}{cccc}
\hline \hline \multicolumn{4}{c}{ Kinematic Range A } \\
$x$ & $y$ & $z$ & $P_{h \perp}[\mathrm{GeV}]$ \\
$0.023-0.27$ & $0.3-0.85$ & $0.2-0.75$ & $0.05-1.0$ \\
\multicolumn{4}{c}{ Kinematic Range B } \\
$x$ & $y$ & $z$ & $P_{h \perp}[\mathrm{GeV}]$ \\
$0.042-0.27$ & $0.3-0.7$ & $0.2-0.6$ & $0.2-0.7$ \\
\hline \hline
\end{tabular}

\section{RESULTS FOR FIXED KINEMATIC RANGES}

The fully differential moments provide the maximum information from this measurement. In order to gain a qualitative picture of the behavior of the moments, a projection to one dimension $(1 D)$ was performed by a weighted integration of the moments over three variables, highlighting the dependence of the moments on the fourth variable. In order to achieve an integral over the selected kinematic ranges, the moment in each kinematic bin is folded with the $\phi$-integrated unpolarized semi-inclusive DIS cross section in this bin, normalized to the same value integrated over the whole kinematic range of the projection. The necessary input cross section for this integration was extracted directly from HERMES data [62].

As anticipated in section $\mathrm{V}$, it is not possible to make a measurement in every bin. Therefore, restricted kinematic ranges of integration were chosen to minimize the number of bins in the integration where a measurement is not possible. In addition, the $z$-bin from 0.75 to 1.0 is excluded as a large fraction of events in this kinematics range contain decay products of exclusively produced hadrons, for which standard factorization might be broken. Hadron and pion results are integrated over the kinematic range $A$, given in table III. This table also lists the reduced kinematic range $B$ used for kaons, which have comparatively lower statistical precision. The average kinematic values for each integrated bin for the ranges $A$ and $B$ are shown in Figs. 2 and 3, respectively. The strong correlation between $x$ and $Q^{2}$ (and thus between $x$ and $y$ ) shown in the bottom left subplot is due to the Hermes acceptance. The other kinematic variables are weakly correlated.

In the integration, bins with large statistical uncertainties (larger than unity) are not included as they do not provide meaningful information to the integral and inflate its uncertainty. The effect of excluding these bins is estimated and included in the systematic uncertainty of the projected results, as described at the end of this section. In figures that compare results from various hadron types or charges, the ranges of integration have been chosen so that only the bins that provide a measurement in the statistically poorest data set are included. This assures 


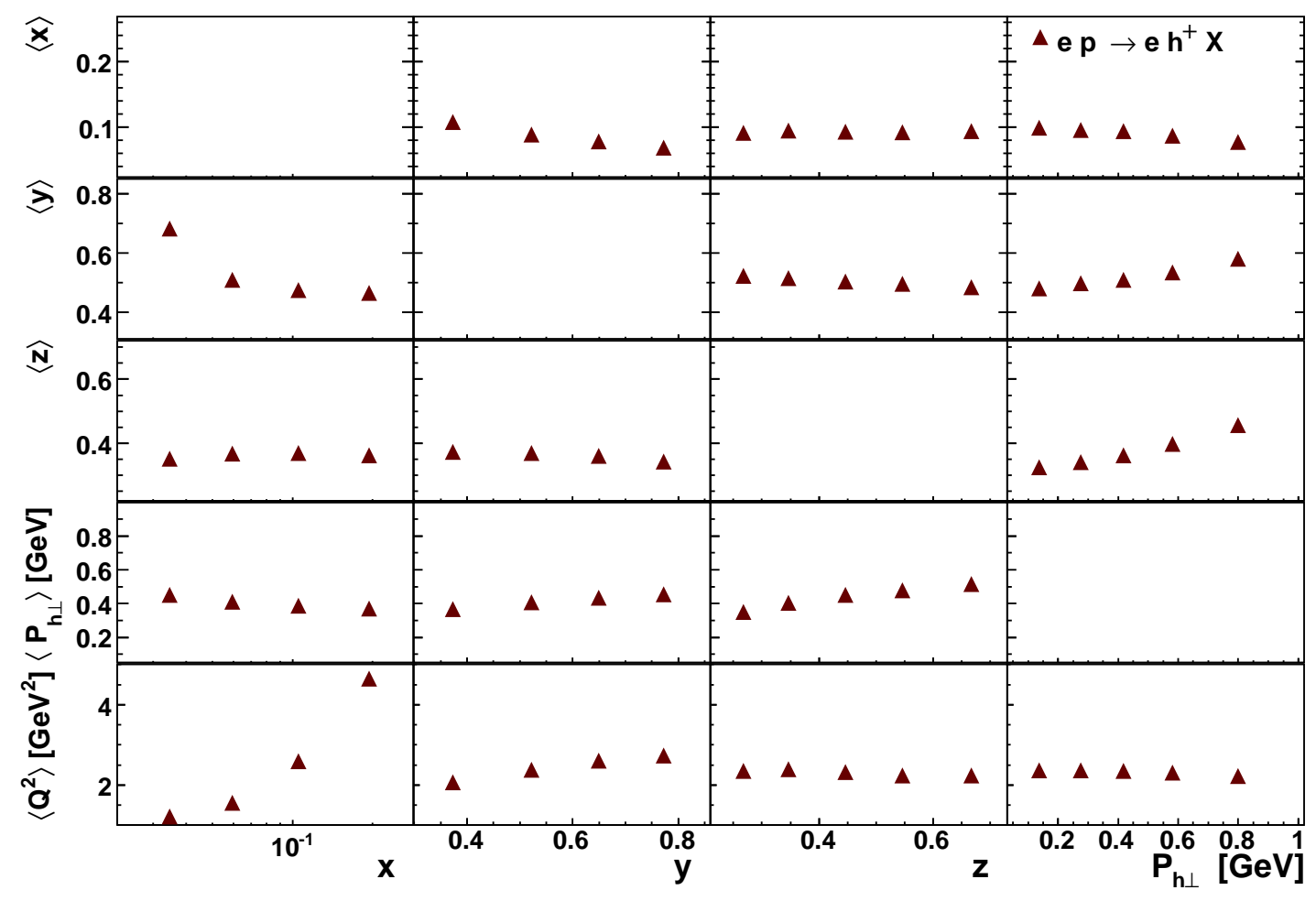

FIG. 2. Average kinematics for the integration range $A$ of table III, as extracted from a $4 \pi$ Monte Carlo (shown here for positive hadrons on hydrogen; other cases exhibit only minor deviations).

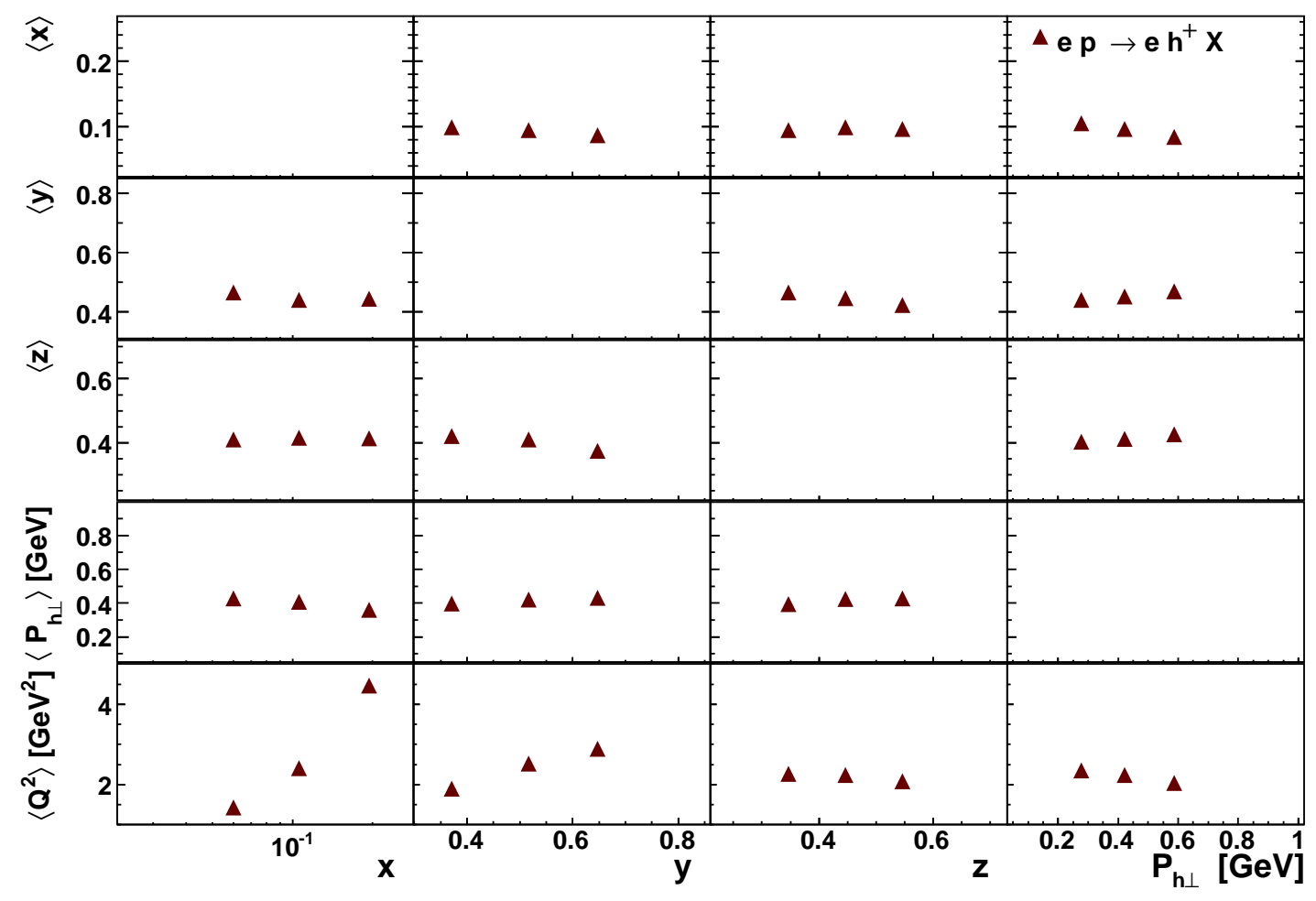

FIG. 3. As in Fig. 2, but for the kinematic range $B$ of table III. 
that the data sets have identical integration regions and thus allows for the results to be compared in a consistent way.

The integrated azimuthal modulations were found to be weakly sensitive to the semi-inclusive DIS cross section used for the integration. This sensitivity was assessed by using the cross section extracted from HeRMES data as well as the cross sections implemented in two Monte Carlo simulations tuned to reproduce the HeRMES measured yields: Pythia6 [53] and GMC_TRAns. The Hermes GmC_trans generator uses the Cteq6 distribution functions [5], and the DSS fragmentation functions $[32,63]$ with the $P_{h \perp}$-dependence based on a Gaussian ansatz. In particular, the transverse momenta $p_{T}$ have a non-constant $z$-dependence as observed from a fit to Hermes data [64], while for Pythia6 this $z$ dependence is flat. The sensitivity to semi-inclusive DIS cross section used for the integration has been added to the systematic uncertainty.

The systematic uncertainties for the results projected in $1 D$ are composed of the uncertainties discussed in section IV, the sensitivity to the cross section used in the integration described in the previous paragraph, plus the additional uncertainty added by excluding some bins from the projection. The Monte Carlo production modified to reproduce the measured azimuthal distribution (described in section IV A) was used to evaluate the effect of the bins excluded from the integration. The difference between including or excluding these bins in the integration of the simulated moments was added to the other systematic contributions. Each systematic contribution was independently projected onto the single variable before the smoothing described in section IV C. After the projection, the systematic contributions were smoothed with a $1 D$ linear fit and then added in quadrature.

\section{A. Results for charged pions}

The cosine modulations for charged pions, projected in the kinematic range $A$ (table III), are presented in this section. All pion samples are projected only including bins that provide a measurement in every data sample, which restricts the integration to those bins with a measurement in the statistically poorest data sample, i.e., the sample for negative pions produced from the deuterium target.

\section{Pion $\cos 2 \phi$ amplitudes}

Figure 4 shows the $\cos 2 \phi$ amplitudes $2\langle\cos 2 \phi\rangle_{U U}$ for pions extracted from hydrogen and deuterium data, projected versus $x, y, z$, and $P_{h \perp}$. Different magnitudes and opposite signs of the amplitudes are observed for oppositely charged pions. In particular, positive $\cos 2 \phi$ amplitudes are extracted for negative pions, while for positive pions the moments are compatible with zero, but tend to be negative in some kinematic regions. The amplitudes for positive and negative pions also exhibit different kinematic dependences. This is particularly evident in their dependence on $z$ : in the integrated kinematic region presented here, the magnitudes for positive pions have no clear kinematic dependence, while they rise with $z$ for negative pions. The amplitudes increase in magnitude with $P_{h \perp}$ for both $\pi^{+}$and $\pi^{-}$, but with opposite signs.

Up to a kinematic suppression of $(1 / Q)$, the $\cos 2 \phi$ amplitudes only contains a single, unsuppressed term, the Boer-Mulders-Collins effect, i.e., the convolution of the Boer-Mulders distribution function $h_{1}^{\perp}\left(x, p_{T}^{2}\right)$ and the Collins fragmentation function $H_{1}^{\perp}\left(z, k_{T}^{2}\right)$ discussed in section I. For a hydrogen target, scattering off up quarks is expected to dominate the reaction ( $u$-dominance), both because the proton consists of more up quarks than down quarks, and because the elementary lepton-quark cross section is proportional to the squared quark electric charge $\left(e_{q}^{2}\right)$, which gives an additional factor of 4 for up quarks compared to down quarks. The Collins function was recently found to have a similar magnitude but opposite sign for fragmentation of up quarks into positive (favored fragmentation) and negative pions (disfavored fragmentation) [65-69]. This would result in different signs for pions of opposite charge, which is in agreement with the data.

The similarity between hydrogen and deuterium results seems to indicate that the Boer-Mulders distribution function has the same sign for up and down quarks, as shown in Ref. [70] and in Ref. [71], and anticipated in Refs. [72, 73]. Although they are similar, for positive pions the deuterium results seem to be systematically closer to zero with respect to the hydrogen results; this might be due to a different magnitude of the BoerMulders function for up and down quarks.

Model calculations [74-77] of the contribution of the Boer-Mulders-Collins effect to the $\langle\cos 2 \phi\rangle_{U U}$ moment are in qualitative agreement with the moments reported here. In particular, the opposite sign for oppositely charged pions seems to be a signature of the Collins effect.

Equation 3 only includes terms up to a suppression $(1 / Q)$, but at a suppression of $(1 / Q)^{2}$ there is at least one additional term that includes the Cahn effect (see Eq. 4). The restricted $Q^{2}$ range of the Hermes data does not allow for a conclusive study that disentangles the leading term from the suppressed terms. Nonetheless, an attempt to describe Hermes results in a more complete way has been done in Ref. [76], where the authors evaluated this suppressed Cahn contribution to the $\cos 2 \phi$ amplitude, assuming a flavor-blind Cahn term, i.e., a flavorindependent $\left\langle p_{T}^{2}\right\rangle$. The comparison of this calculation to data indicates that, in the HeRMEs kinematic regime, the Cahn term is smaller than expected or is counteracted by additional terms that have been neglected. In the same paper, a possible Cahn flavor-dependence was also estimated by varying the $\left\langle p_{T}^{2}\right\rangle$ for down quarks while maintaining a fixed $\left\langle p_{T}^{2}\right\rangle$ for up quarks; no significant changes 
were observed in the calculated Cahn term. However, this test was performed on a hydrogen target, and not a deuterium target where the results might be more sensitive to the down quarks.

In Ref. [71], the authors attempted to simultaneously describe preliminary unidentified hadron $\cos 2 \phi$ amplitude extracted at HERMES [78] and COMPASs [39]. The Boer-Mulders-Collins effect is described using the Collins fragmentation function from Ref. [66] while for the Boer-Mulders function, the same functional form that was used for the Sivers function [79] was applied. In the calculation the Cahn effect is also included, which is sensitive to the quark average transverse momenta. The previously reported average momentum of $\left\langle p_{T}^{2}\right\rangle=0.25 \mathrm{GeV}^{2}[80]$ describes the CompAss data well. In contrast, the HERMES data is better described by the lower value of $\left\langle p_{T}^{2}\right\rangle=0.18 \mathrm{GeV}^{2}$, leading to a smaller Cahn effect at Hermes. This is in accordance to the broadening of the $p_{T}$ distribution when considering $Q^{2}$ evolution, as observed in Ref. [81].

\section{Pion $\cos \phi$ amplitudes}

The $\cos \phi$ amplitudes come suppressed as $1 / Q$ in the hadron cross section, and, in contrast to the $\cos 2 \phi$ amplitudes, several terms contribute at same level of suppression (Eq. 5). Results for the $\cos \phi$ amplitudes $2\langle\cos \phi\rangle_{U U}$ extracted for pions from hydrogen and deuterium data are shown in Fig. 5. Results extracted from hydrogen and deuterium are similar, but deuterium results for positive pions are smaller than hydrogen results. This could be related to flavor dependence of the contributions involved in the amplitudes. The $\cos \phi$ amplitudes are found to be negative for both positively and negatively charged pions, but for positive pions they are in general larger in magnitude. For both positive and negative pions, the magnitudes increase with the pion energy fraction $z$.

The $z$ dependence of the amplitudes can be interpreted in terms of the Cahn effect. Indeed, Cahn anticipated a rise of amplitudes with $z$ due to the reduced dilution by the random transverse momentum that the pions acquire during fragmentation $[17,18]$. At high $z$ the amplitudes for oppositely charged pions are very similar and reach their largest magnitude (up to -0.2). Different behaviors are observed for oppositely charged pions versus $P_{h \perp}$. The magnitude of the amplitudes for positive pions increases with $P_{h \perp}$, supporting the Cahn expectations of a signal proportional to transverse momentum. But, this trend is not observed for negative pions, suggesting that contributions to Eq. 5 other than Cahn possibly counterbalance the increase with $P_{h \perp}$. The Cahn term is expected to be weakly sensitive to flavor, as discussed in the previous section. As in the case of the $\cos 2 \phi$ amplitudes, the difference between oppositely charged pions can be generated by flavor dependent contributions, like, e.g., the Boer-Mulders-Collins effect.

In contrast to the $\cos 2 \phi$ amplitudes, no model can qualitatively describe the measured amplitudes for $\cos \phi$. To date, only one prediction has been published for the Hermes $\cos \phi$ amplitude [82], which includes the Cahn term only. The amplitudes predicted are larger than the measurements, suggesting that the Cahn contribution at HERMES is smaller than expected. As in the case of $\langle\cos 2 \phi\rangle_{U U}$, at least part of the discrepancy can be related to a $\left\langle p_{T}^{2}\right\rangle$ that, in HERMES kinematic conditions, is smaller than $0.25 \mathrm{GeV}^{2}$. Moreover, the modeled Cahn term cannot describe the observed difference between $\pi^{+}$and $\pi^{-}$, as it was assumed to be flavor-blind. This implies that for a qualitative description of the measured $\cos \phi$ amplitudes, a more complex Cahn contribution, or additional flavor dependent contributions, like, e.g., the Boer-Mulders-Collins effects, must be taken into account. Furthermore, in addition to the Cahn and the Boer-Mulders terms, the structure function $F_{U U}^{\cos \phi}$ includes four terms related to quark-gluon-quark correlators which have not been taken into account in this interpretation of the data, as little is known about the underlying physics.

\section{B. Results for charged kaons}

This section presents the cosine modulations extracted for charged kaons, projected in the reduced kinematic range $B$ of table III. All kaon samples are projected using bins that provide a measurement in the negative kaon sample produced from the deuterium target, which is the statistically poorest kaon data sample. No model calculation for kaons is available to date.

\section{Kaon $\cos 2 \phi$ amplitudes}

The $\cos 2 \phi$ amplitudes extracted for charged kaons are shown in Fig. 6 for the hydrogen and deuterium targets. The amplitudes are large in magnitude (up to -0.1), and have the same negative sign for both positive and negative kaons, in contrast to the trends observed for pions. This may be interpreted by considering the kaon's quark content: the valence quark content of $K^{+}$mesons is $u \bar{s}$, and therefore $K^{+}$production is expected to receive a large contribution from lepton scattering off up quarks (u-dominance). A favored Collins fragmentation function is expected to be involved in this case, as in the case of $\pi^{+}$. In the framework of the Artru model [83], all favored Collins functions describing fragmentation into spin-zero mesons have the same sign. Therefore, the Boer-Mulders-Collins effect for positive pions and kaons is expected to have the same sign, as observed in measurements. Nothing is known about the Collins fragmentation function into kaons. A significant contribution from sea quarks cannot be excluded. For example, strange quarks may contribute at $x<0.1$ [84], as suggested by unpolarized fragmentation, where the strange quark fragmentation function into $K^{+}$appears to be sig- 


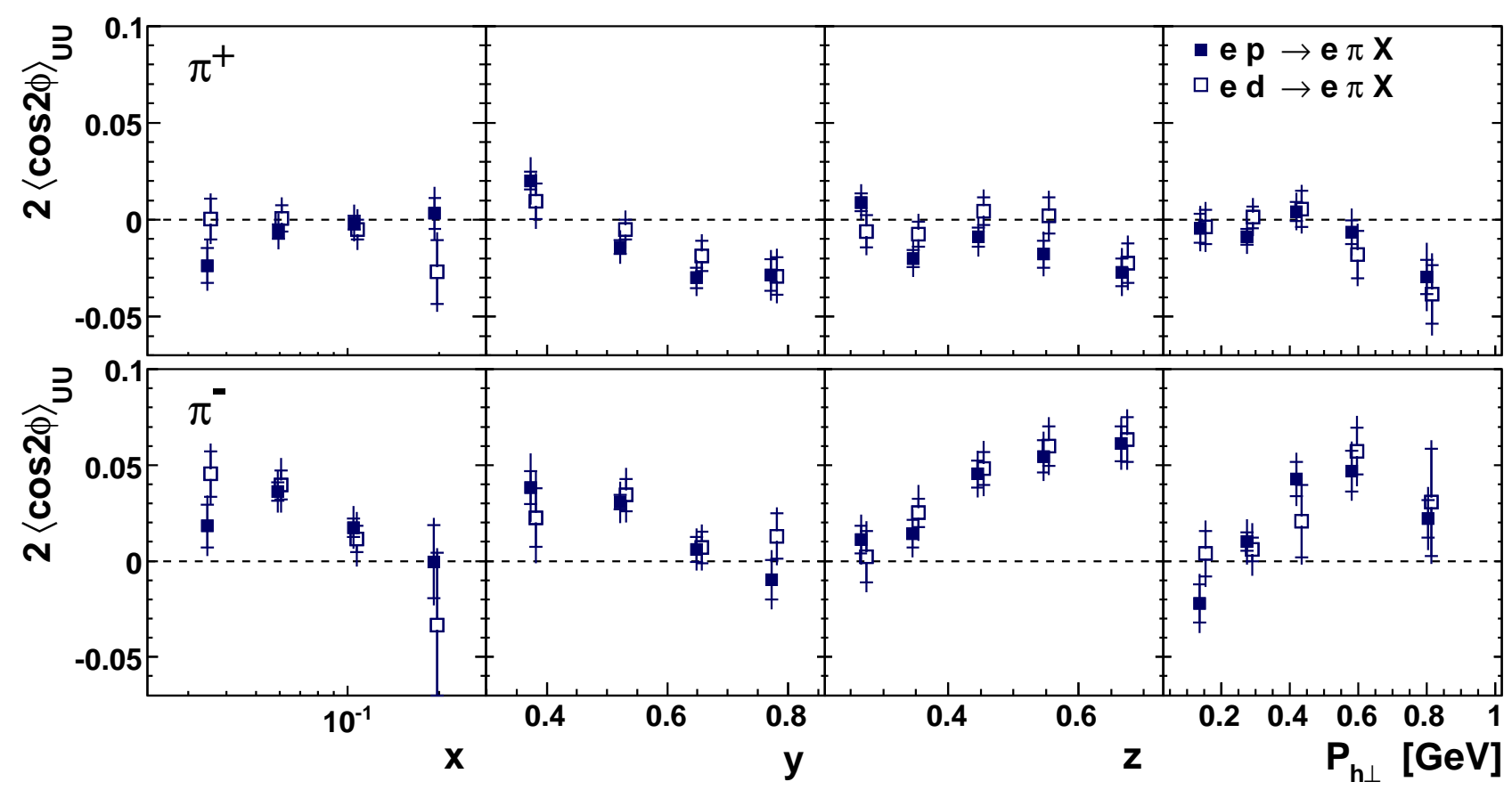

FIG. 4. $\cos 2 \phi$ amplitudes for positive (upper panels) and negative (lower panels) pions integrated over the kinematic range $A$ of table III. Closed and open squares are for amplitudes extracted from hydrogen and deuterium targets, respectively. The inner bar represents the statistical uncertainty; the outer bar is the total uncertainty, evaluated as the sum in quadrature of statistical and systematic uncertainties. Points have been slightly shifted horizontally for visibility.

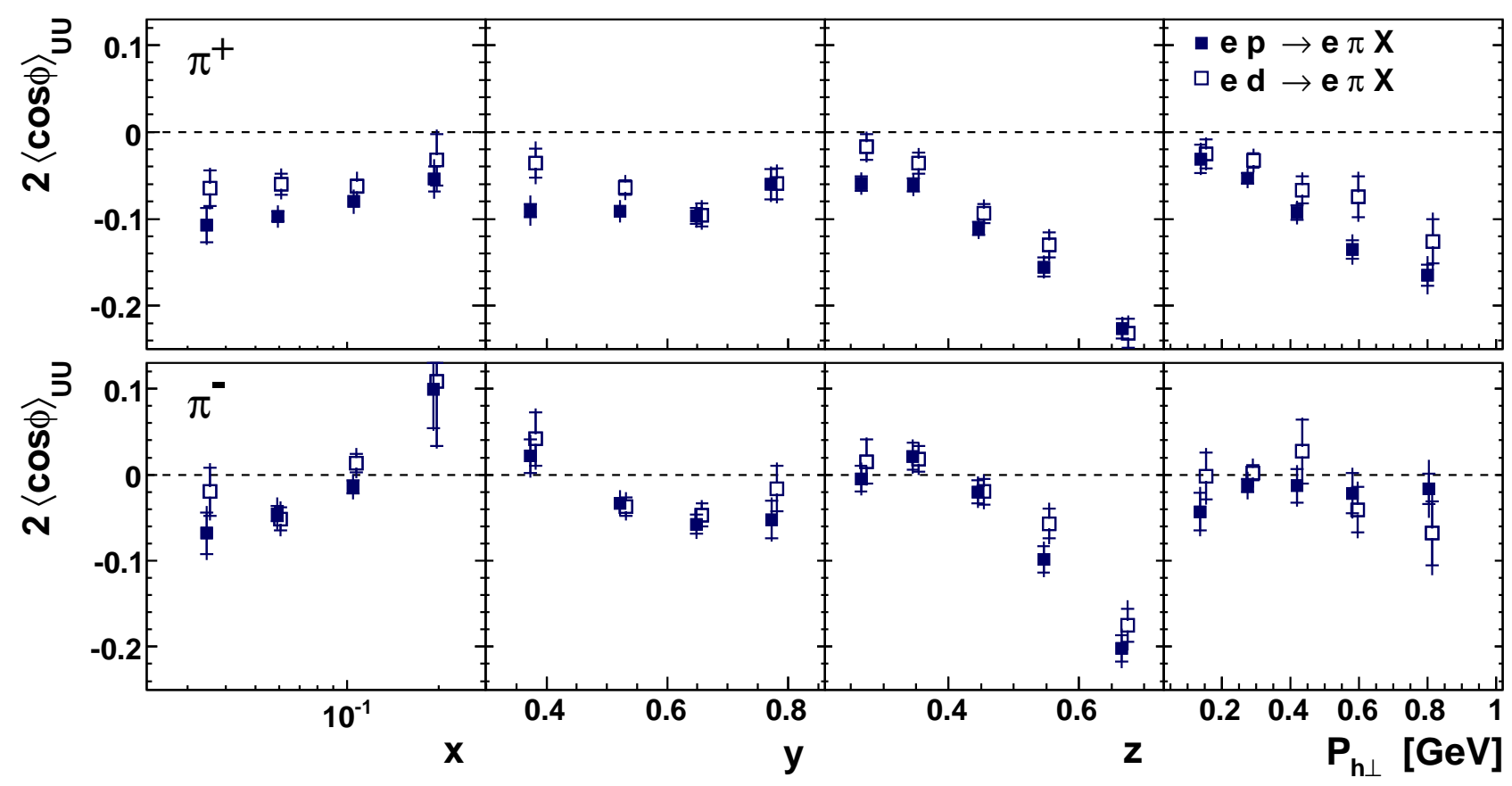

FIG. 5. As in Fig. 4, but for the $\cos \phi$ amplitudes. 
nificantly larger than the fragmentation function for up quarks into $K^{+}$[32]. A substantial difference between the strange and the up and down Collins fragmentation functions would play an important role in the observed moments.

For negative kaons the situation is even more complicated, as its valence quark content $(s \bar{u})$ does not include any quarks in common with the valence structure of the target. Therefore, even larger contributions can be expected to originate from the sea and from disfavored up quark fragmentation.

Similar kaon amplitudes are extracted from hydrogen and deuterium targets. This may reflect similar contributions from $u$ and $d$ quarks, as well as a potentially substantial contribution from strange quark fragmentation, which is expected to be the same for neutron and proton targets. Contrary to pions, the positive kaons show hydrogen results closer to zero, which might reflect a different magnitude for the Boer-Mulders function of different quark types, or the increased role of disfavored up quark fragmentation for proton targets.

\section{Kaon $\cos \phi$ amplitudes}

The $\cos \phi$ amplitudes for kaons are shown in Fig. 7 for hydrogen and deuterium targets. Large negative (up to -0.2) amplitudes are extracted for positive kaons, slightly rising with $z$ and $P_{h \perp}$. The amplitudes are even larger in magnitude than those for positive pions, which suggests a large contribution from the Boer-MuldersCollins effect, which was found to be large for $K^{+}$in the previous section. Negative kaons instead show results compatible with zero. The similarity between the $\cos 2 \phi$ amplitudes for positive and negative kaons may mean that the Boer-Mulders-Collins effect is relatively insensitive to kaon charge. Thus, the significant difference in the $\cos \phi$ amplitudes for positive and negative kaons points to either a flavor dependence of the Cahn contribution (e.g. from strange quarks) or a significant contribution from the interaction dependent terms that have been otherwise neglected in this discussion. Similar results are extracted for scattering off hydrogen and deuterium.

\section{Results for unidentified charged hadrons}

In this section the cosine modulations extracted for unidentified hadrons and projected in kinematic range $A$ (table III) are presented. As for identified charged hadrons, individual kinematic bins are included in the integration only if they provide a measurement in the statistically poorest unidentified hadron data sample, i.e., negative hadrons produced from a deuterium target.

As the majority of the unidentified hadrons consists of pions ( $\gtrsim 70-88 \%$, depending on the hadron's charge), the amplitudes of unidentified hadrons are very similar to those of pions, and most of the arguments from the discussion of the pion results also apply here. However, as no hadron identification was required, the systematic uncertainty for the unidentified hadron sample does not include a contribution from the RICH identification. The remaining hadrons are in large part kaons $(\sim 10 \%)$, and protons $(\sim 10 \%)$. As no theoretical model has evaluated the cosine modulations for kaons and protons, no predictions exist for the unidentified hadron sample.

\section{Hadron $\cos 2 \phi$ amplitudes}

Figure 8 shows the $\cos 2 \phi$ amplitudes of unidentified hadrons extracted from hydrogen and deuterium data. Different amplitudes are extracted for oppositely charged hadrons; in particular, they are of opposite sign, as in the case of pions. Similar amplitudes are observed for hadrons produced from hydrogen and deuterium targets.

\section{Hadron $\cos \phi$ amplitudes}

Results for the $\cos \phi$ amplitudes extracted from hydrogen and deuterium data are shown in Fig. 9. They are found to be negative for both positively and negatively charged hadrons, but larger in magnitude for the positive hadrons. Hadrons produced using hydrogen and deuterium targets result in similar amplitudes, but small differences can be observed for $h^{+}$that reflect the behavior of the $\pi^{+}$amplitudes.

\section{Comparison of amplitudes for various hadron types}

In order to compare the cosine modulations extracted for the various hadron types, all samples were projected in the smaller integration range $B$ of table III and bins were only included if they provided a measurement in the negative kaon sample produced from a deuterium target, which is the statistically poorest data sample. Figures 10, 11,12 and 13 show the comparisons of the $\cos 2 \phi$ and $\cos \phi$ amplitudes for the various hadron types produced on a hydrogen and deuterium target. The kaon moments are substantially larger in magnitude than those of the pions, with the exception that the $K^{-}\langle\cos \phi\rangle_{U U}$ moments are compatible with those of $\pi^{-}$, although their large uncertainties also make them compatible with zero. The $\langle\cos 2 \phi\rangle_{U U}$ moments for negative kaons not only have a larger magnitude but also the opposite sign as the pion moments. A magnitude of $K^{+}$amplitudes larger than that for $\pi^{+}$was already observed in the case of the amplitudes measured in transverse-target single-spin asymmetries where the Collins fragmentation function couples to the transversity distribution function [67]. The large amplitudes for kaons suggest a Collins effect that 


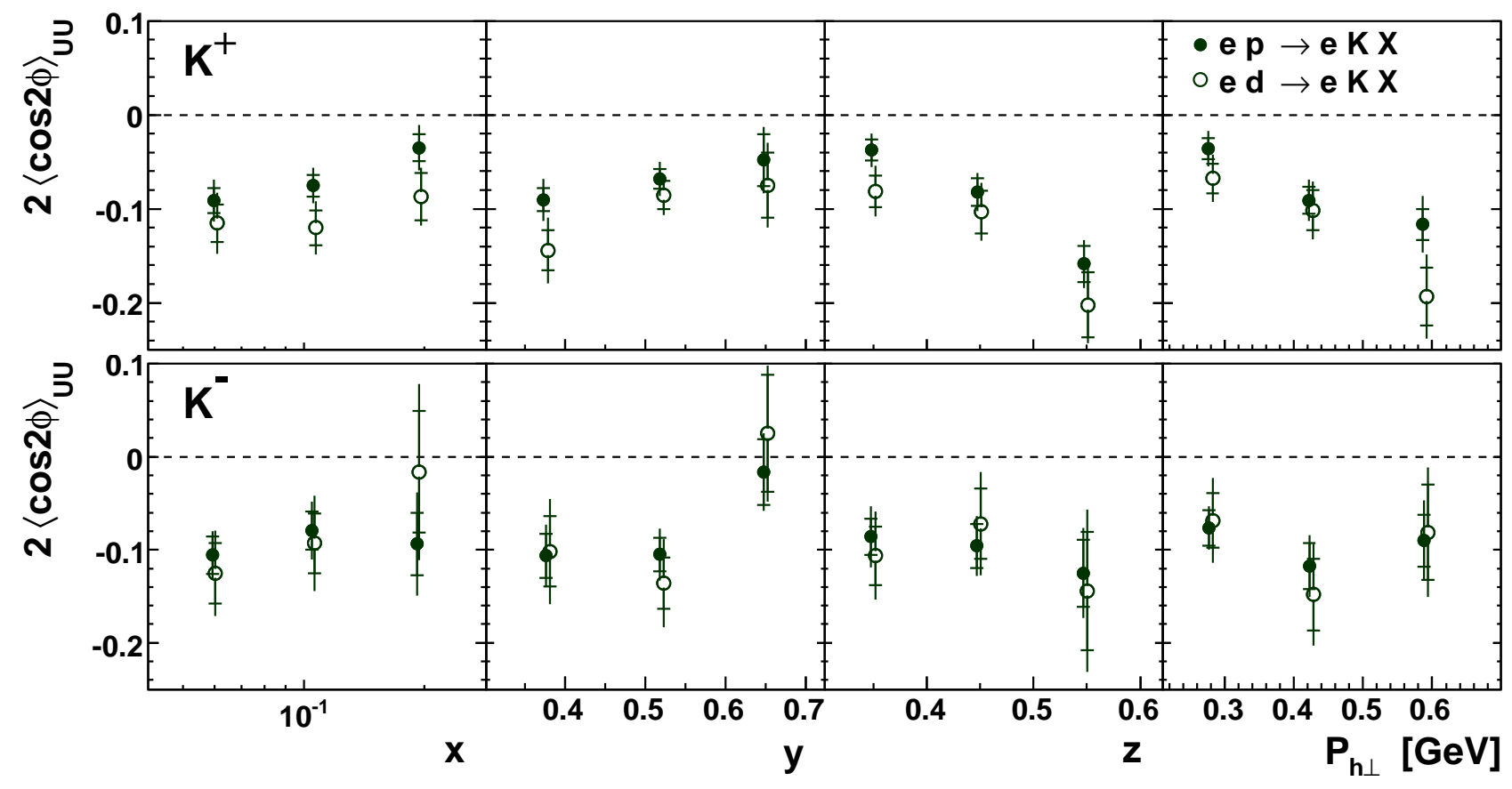

FIG. 6. As in Fig. 4, but for charged kaon amplitudes integrated over the kinematic range $B$ of table III.

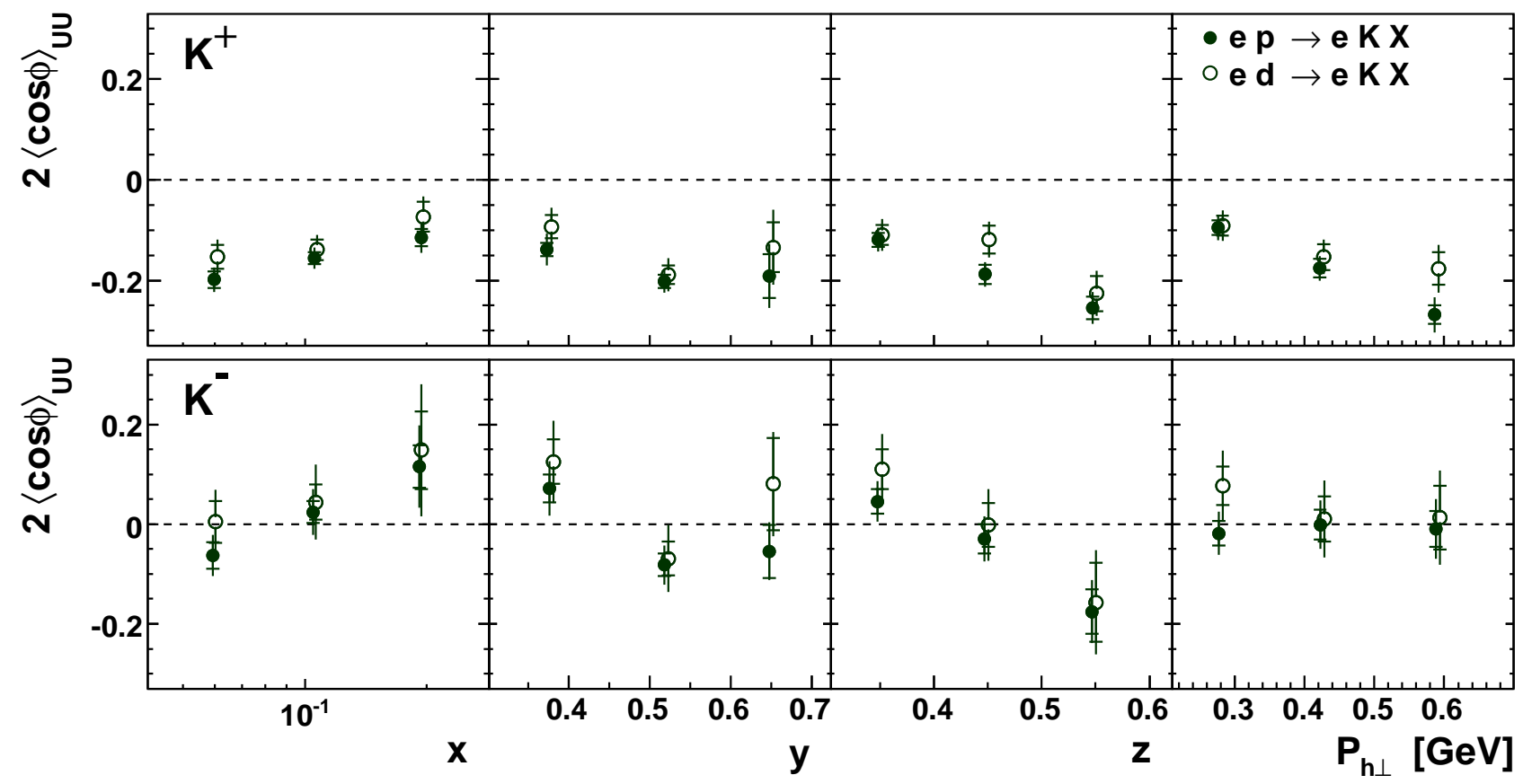

FIG. 7. As in Fig. 5, but for charged kaon amplitudes integrated over the kinematic range $B$ of table III.

is larger for kaons than for pions; in addition, the differences with respect to pions can arise from a significant role of strange quarks in kaon production. The modulations extracted for unidentified hadrons and pions have similar trends, although some differences are observed, particularly for the $\cos 2 \phi$ amplitudes. The discrepancies between hadrons and pions are generally consistent with the observed kaon moments. 


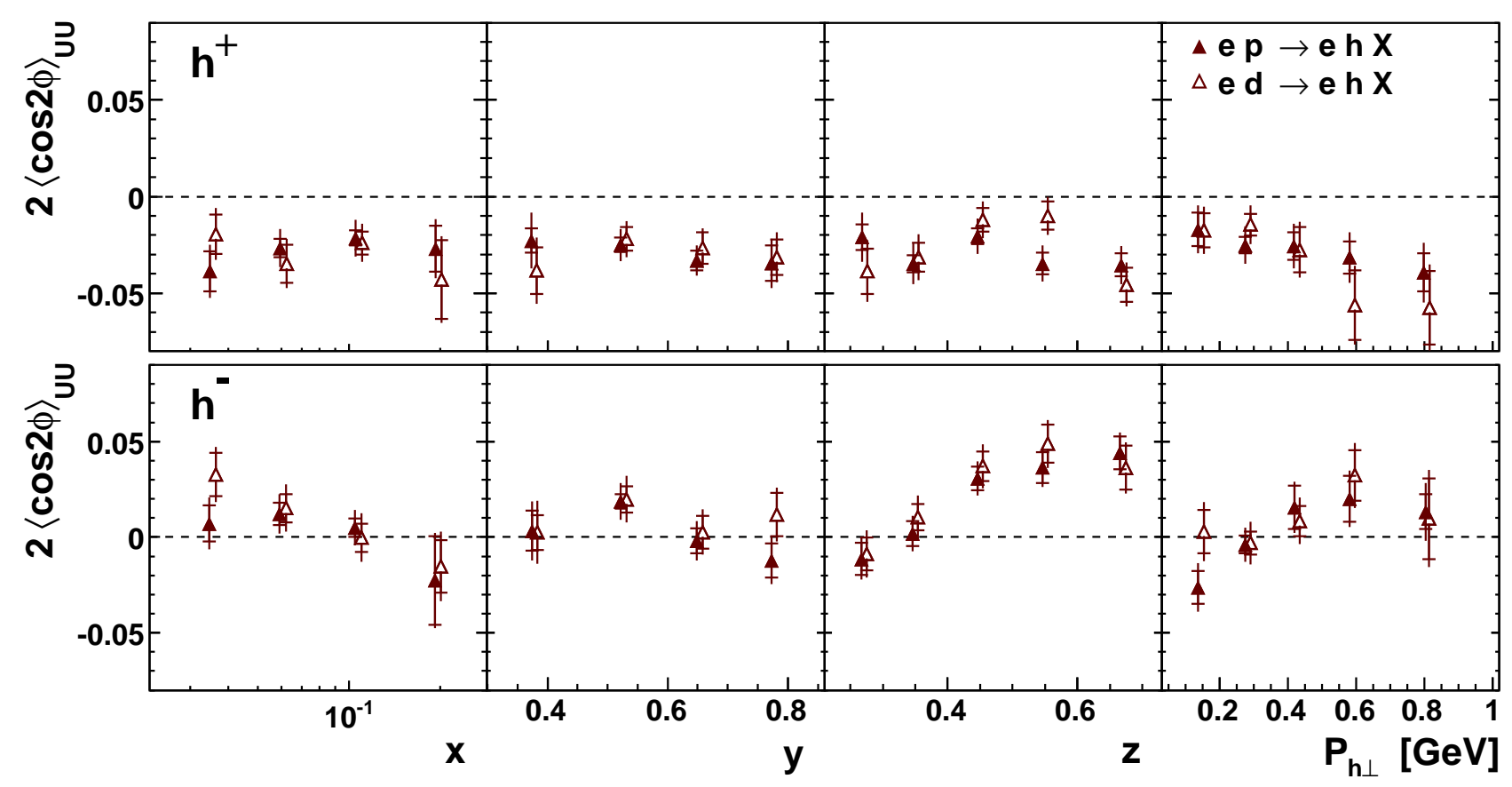

FIG. 8. As in Fig. 4, but for unidentified charged hadrons.

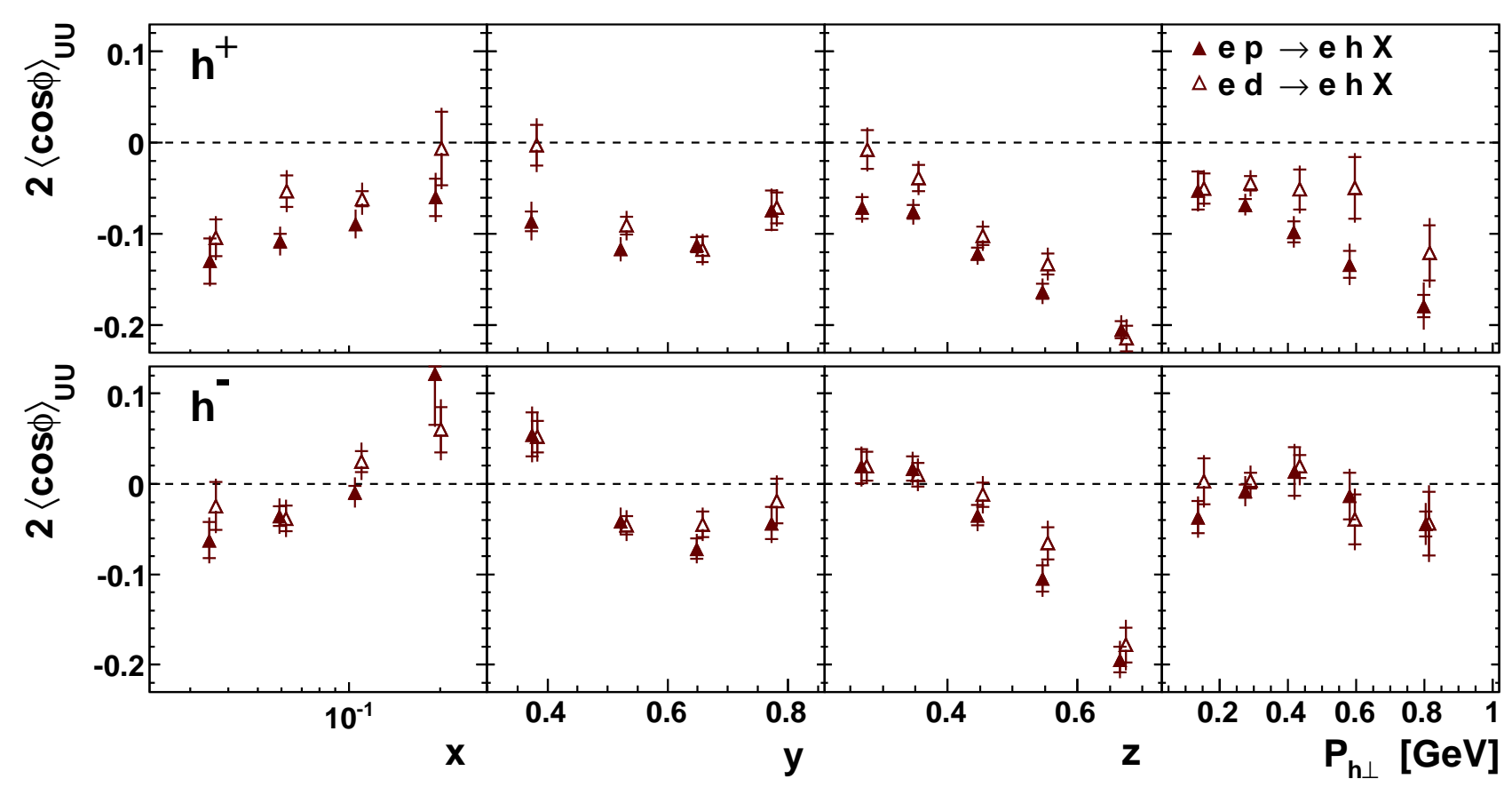

FIG. 9. As in Fig. 5, but for unidentified charged hadrons.

E. Charge difference

The systematic uncertainties of results in Figs. 4-9 are highly correlated for positive and negative hadrons of the same type, as they were measured under the same ex- perimental conditions. It is therefore useful to provide the difference between the amplitudes of positive and negative hadrons, where many systematic uncertainties cancel. The charge difference provides more strict constraints for models, as it accounts for correlated systematics between hadrons of the same type, but different 


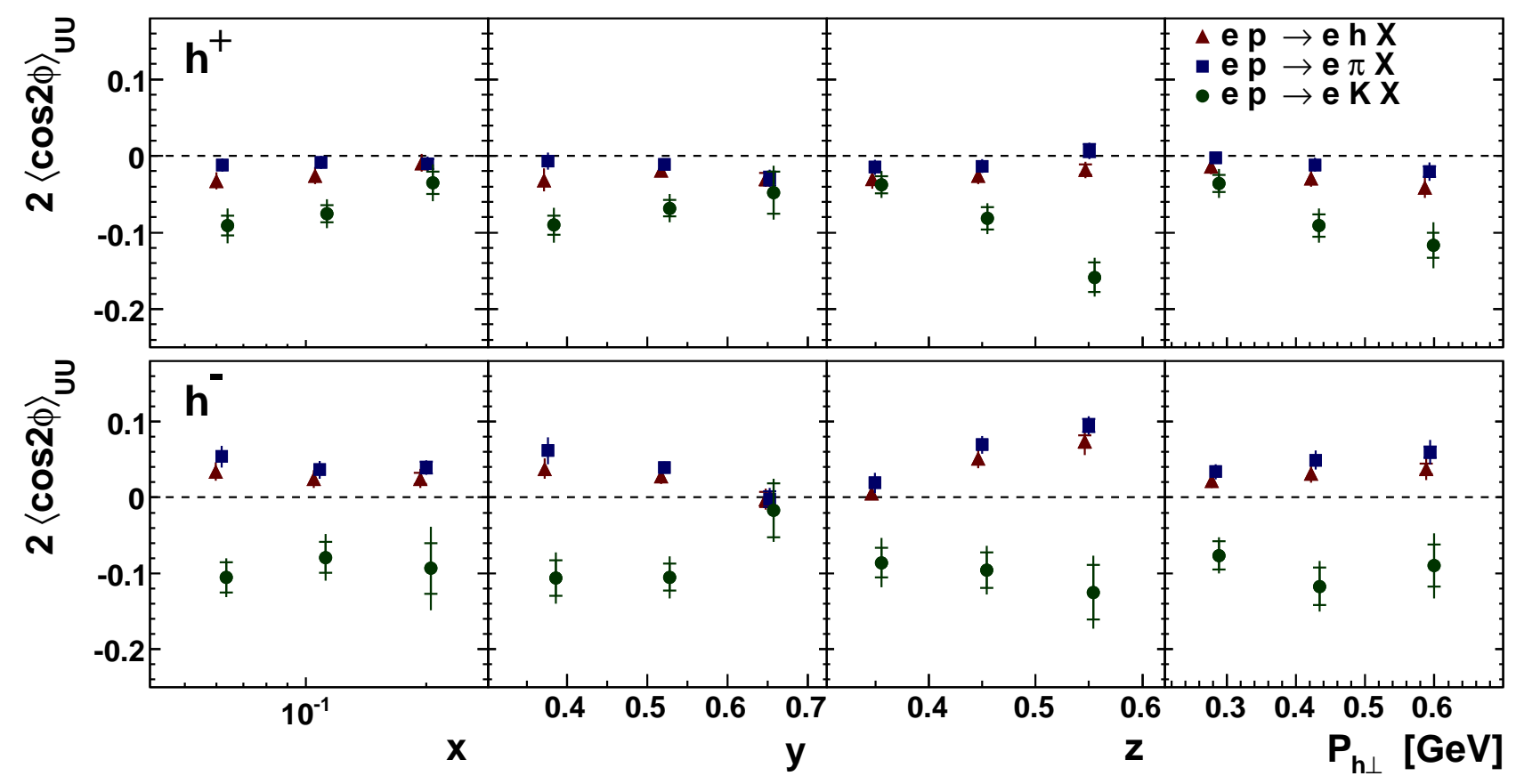

FIG. 10. $\cos 2 \phi$ amplitudes from a hydrogen target for positive (upper panels) and negative (lower panels) unidentified hadrons (triangles), pions (squares) and kaons (circles), integrated over the kinematic range $B$ of table III. Uncertainties as in Fig. 4. Points have been slightly shifted horizontally for visibility.

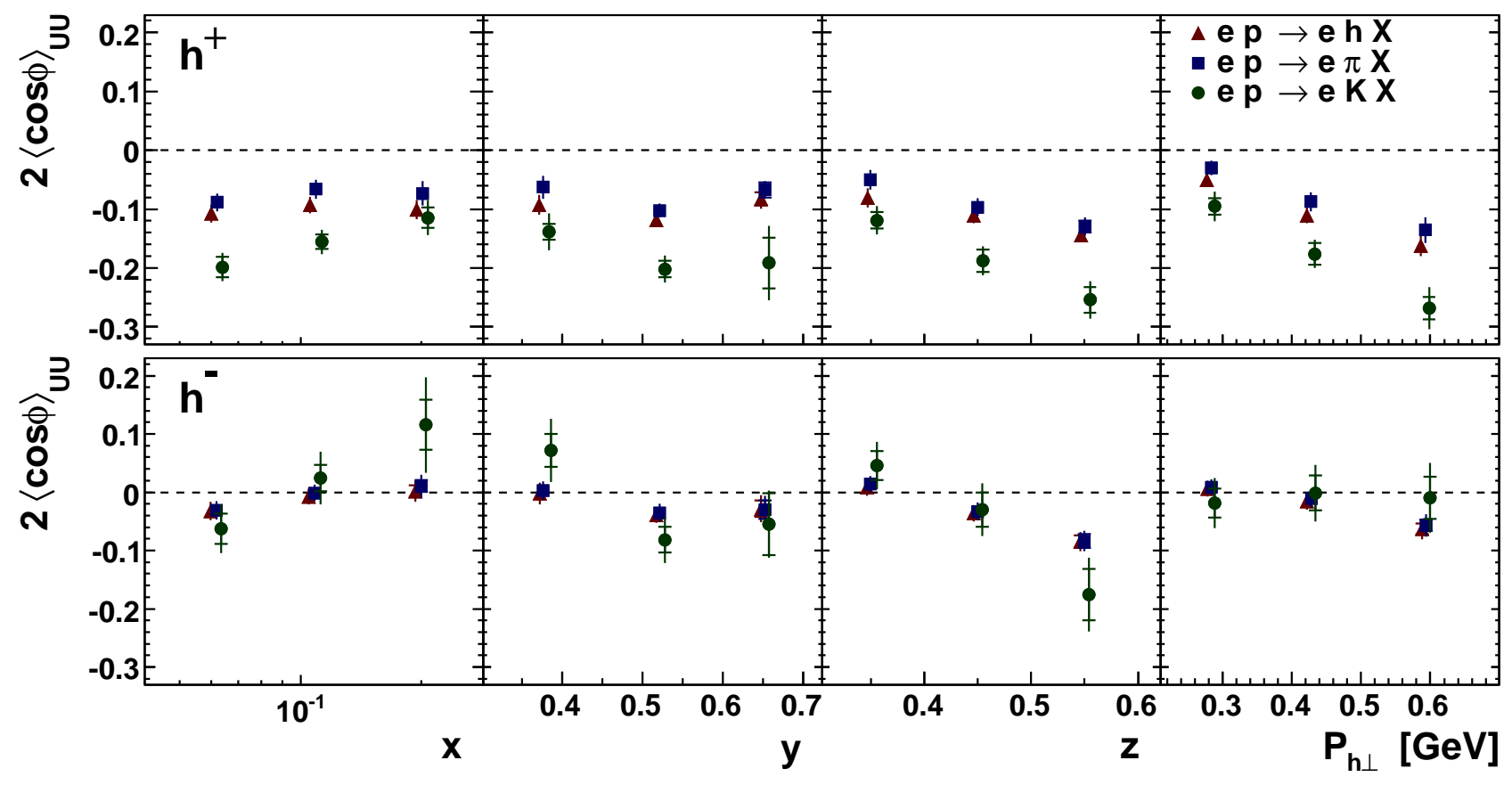

FIG. 11. $\cos \phi$ amplitudes from a hydrogen target for positive (upper panels) and negative (lower panels) unidentified hadrons (triangles), pions (squares) and kaons (circles), integrated over the kinematic range $B$ of table III. Uncertainties as in Fig. 4. Points have been slightly shifted horizontally for visibility. 


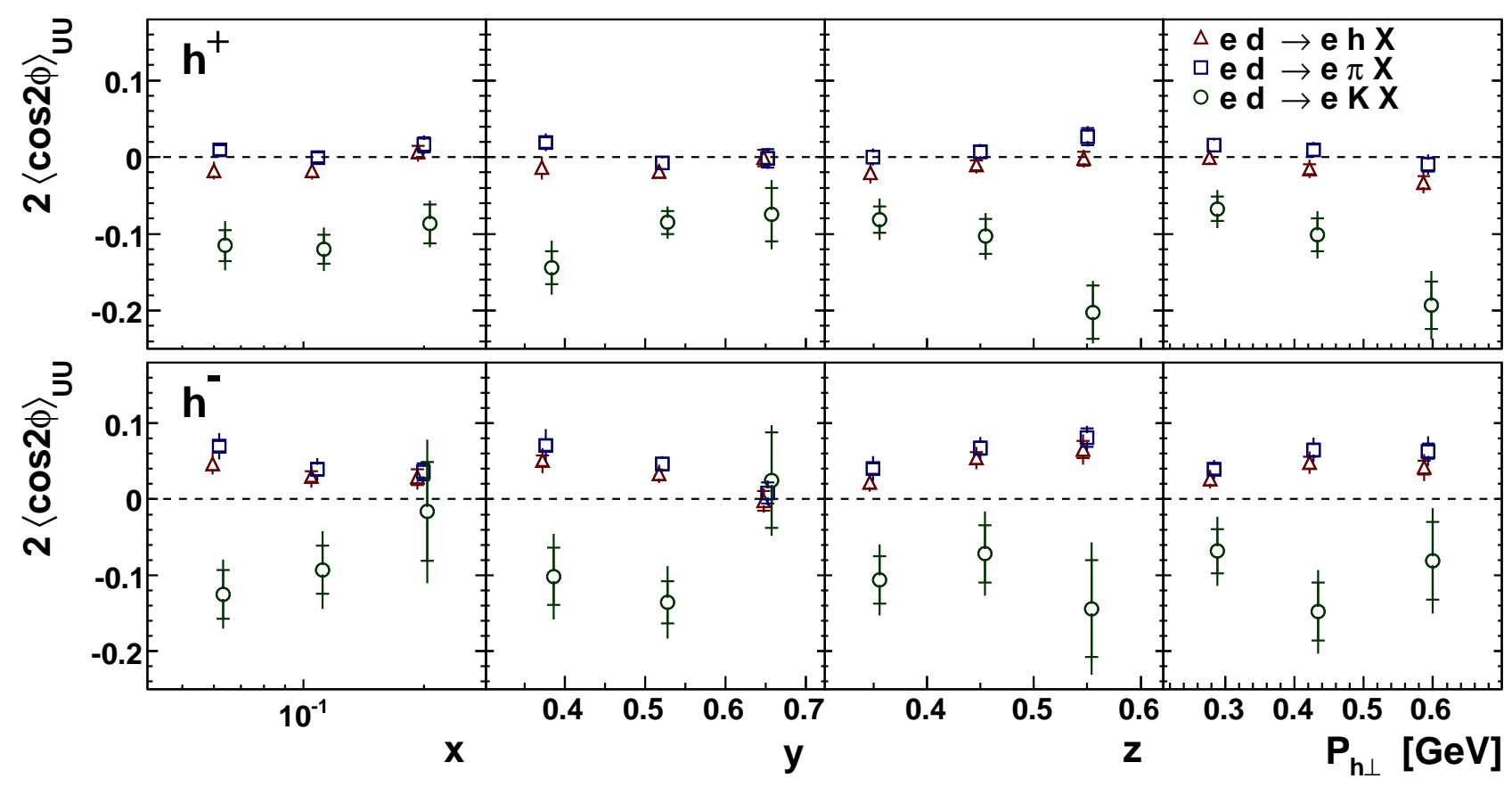

FIG. 12. As in Fig. 10, but for a deuterium target.

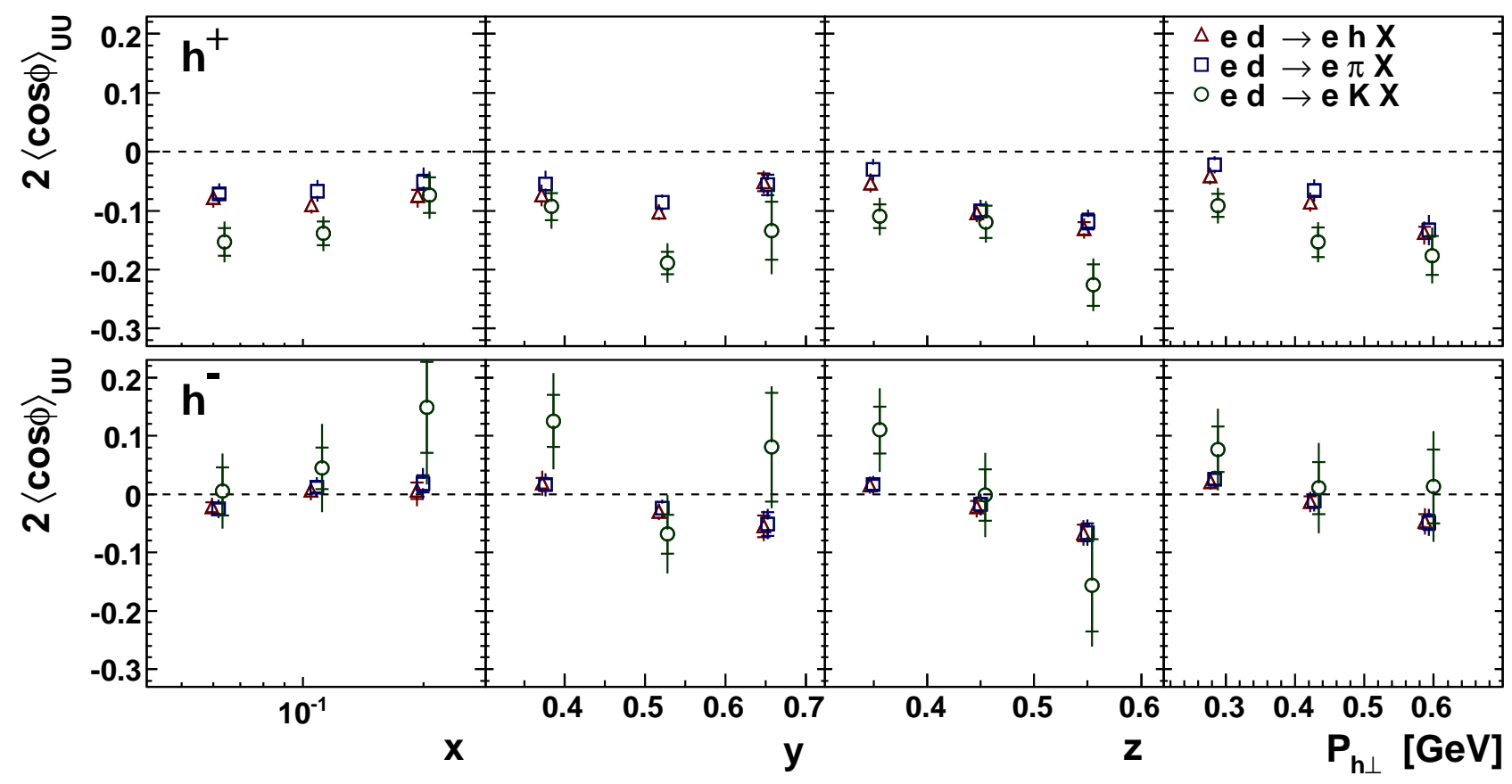

FIG. 13. As in Fig. 11, but for a deuterium target.

charge. In addition some hadron-flavor blind contributions to the moment may be suppressed, e.g., a Cahn effect as considered so far in most phenomenological approaches. In that case, both the $\cos \phi$ and $\cos 2 \phi$ charge difference amplitudes are expected to have an increased sensitivity to the Boer-Mulders-Collins effect.
For each hadron type the charge difference of the respective amplitudes was evaluated, and its uncertainty was computed, taking into account the correlations. The results are shown in Figs. 14, 15, and 16, for pions, kaons, and unidentified hadrons, respectively. For pions and unidentified hadrons the charge difference is significantly 


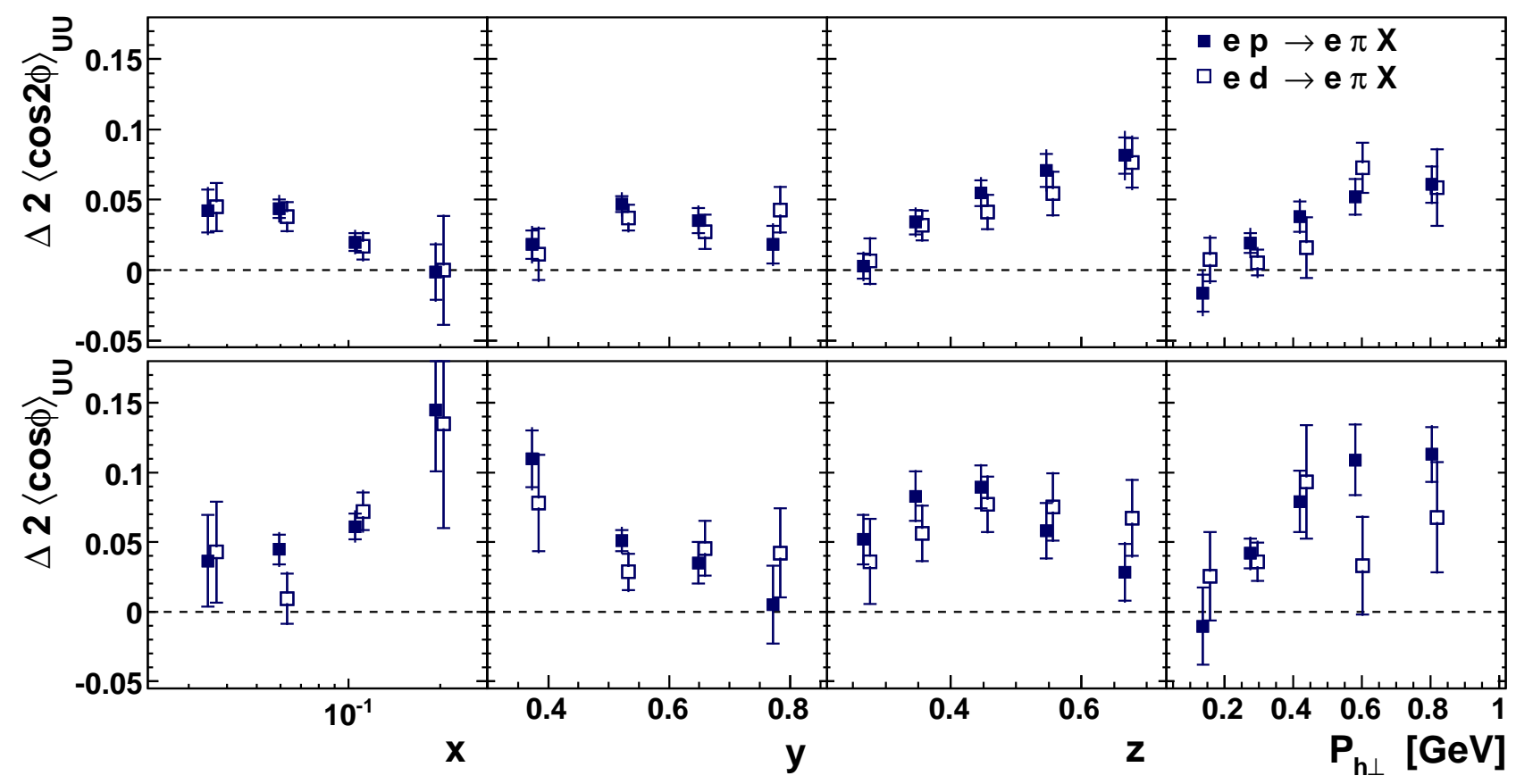

FIG. 14. Difference between the amplitudes of negative and positive pions within the kinematic range $A$ of table III: $2\langle\cos 2 \phi\rangle_{\pi^{-}}-2\langle\cos 2 \phi\rangle_{\pi^{+}}$(upper panels), $2\langle\cos \phi\rangle_{\pi^{-}}-2\langle\cos \phi\rangle_{\pi^{+}}$(lower panels). Closed and open symbols are for amplitudes extracted from hydrogen and deuterium targets, respectively. Points have been slightly shifted horizontally for visibility. Uncertainties as in Fig. 4.

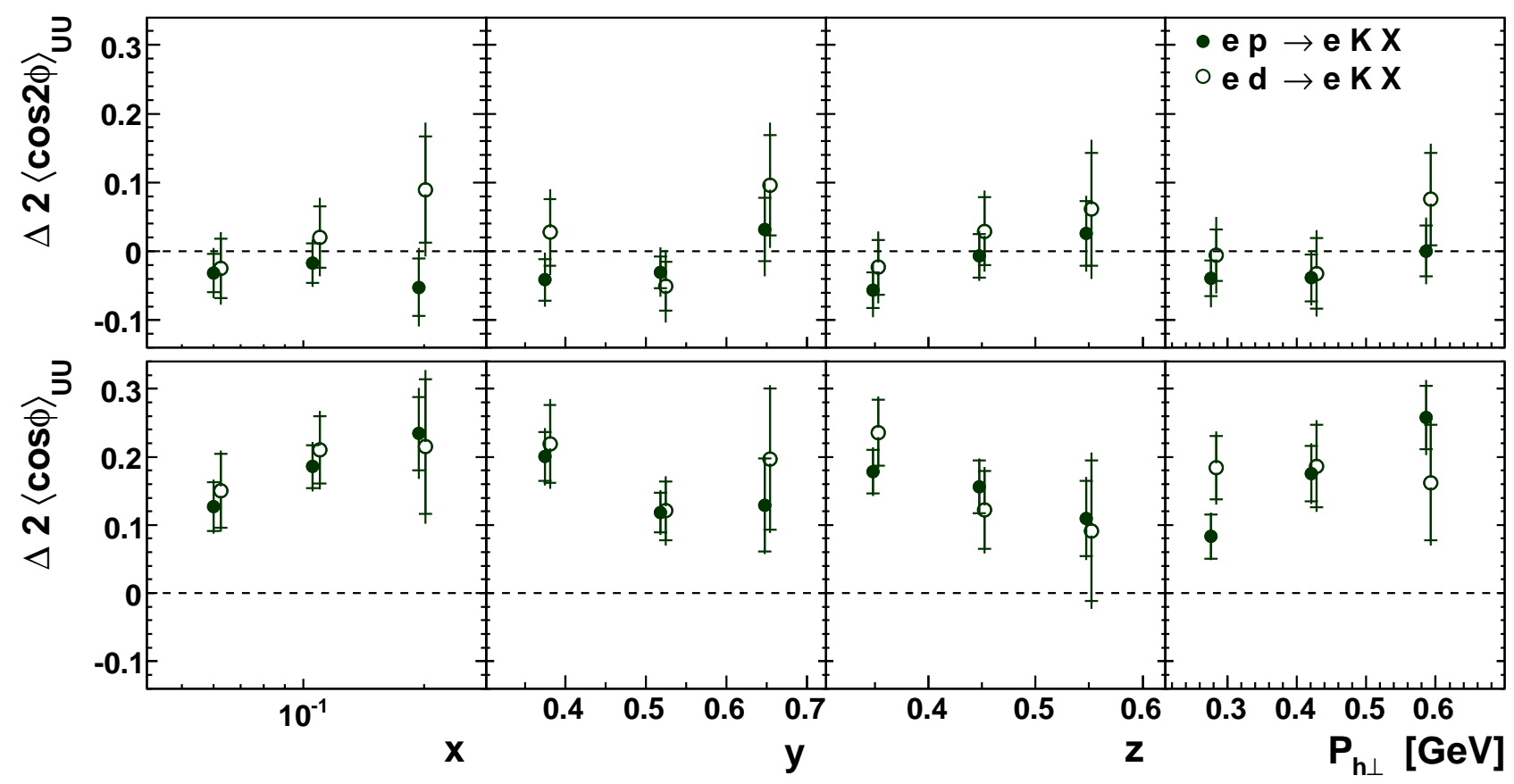

FIG. 15. As in Fig. 14, but for kaons, and the kinematic range $B$ of table III. 


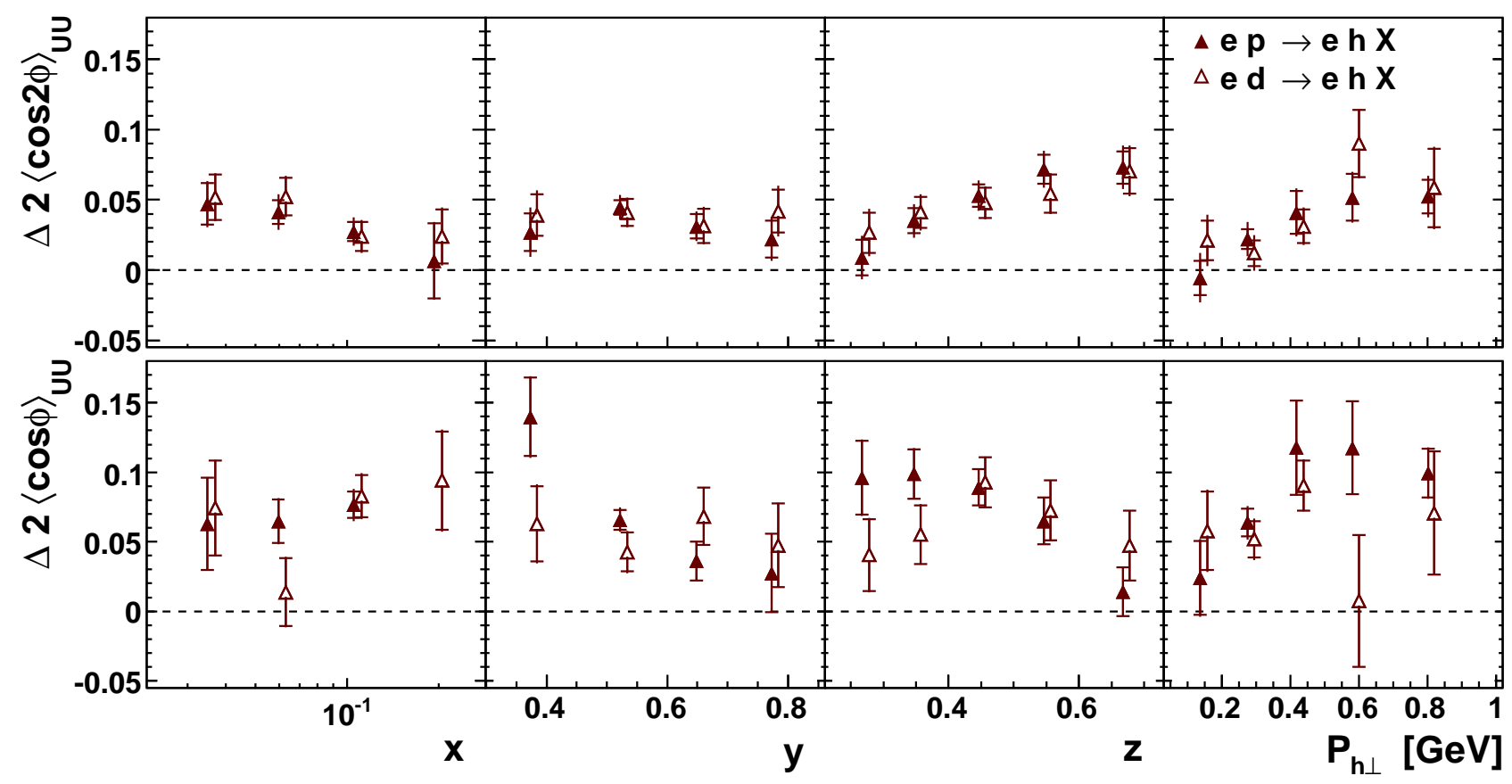

FIG. 16. As in Fig. 14, but for unidentified hadrons.

non-zero over nearly the entire kinematic range. For kaons, a large charge difference is observed for the $\cos \phi$ amplitudes, while the difference for the $\cos 2 \phi$ amplitudes is compatible with zero. The different behavior of kaons with respect to pions suggests an important contribution to the modulations from scattering off strange quarks, or, more generally, from scattering off the sea.

\section{CONCLUSIONS}

HERMES measured fully-differential $(4 D)$ cosine modulations for charged pions, kaons, and unidentified hadrons produced in semi-inclusive DIS off unpolarized hydrogen and deuterium targets. In the TMD framework, these amplitudes can be interpreted by a non-zero intrinsic transverse momentum of quarks (Cahn effect) and by correlations between the quark's transverse polarization and its transverse momentum and the transverse momentum of the produced hadron (Boer-Mulders-Collins effect). However, considering the low average $Q^{2}$ attainable at Hermes, contributions suppressed as $(1 / Q)^{2}$ and higher may be not negligible.

To date, the cosine modulations presented here represent the most complete data set on the subject, and allow access to flavor-dependent information on the internal degrees of freedom of the nucleon. The extracted $4 D$ amplitudes, with their full covariance matrix, provide the maximum information from this measurement, and can be used to guide model construction in a fully differential way.
In addition, the amplitudes have been presented as projections over specific integration ranges of the four kinematic variables $x, y, z$, and $P_{h \perp}$. Significant differences are observed for moments extracted for oppositely charged pions, interpreted as being due to the convolution of the Boer-Mulders and the Collins functions. The extracted amplitudes for pions and kaons show different sizes and kinematic dependences. In particular, the $\cos 2 \phi$ amplitudes for kaons are larger than for pions, and do not change sign for oppositely charged kaons. The former may be due to different features of Collins fragmentation into kaons and pions, possibly related to a significant contribution from strange quarks to kaon production.

The amplitudes extracted from hydrogen and deuterium targets are found to be similar, but slightly different for positive pions and kaons. For pions, a similar size can be due to the Boer-Mulders distribution functions with the same sign for up and down quarks. For kaons this can be due to a similar contribution from up and down quarks along with a similar strange sea distribution in protons and neutrons. The slight differences for hydrogen and deuterium targets for positive pions and kaons might be related to a slightly different magnitude of the Boer-Mulders functions for the different quark types. The difference of moments between positively and negatively charged hadrons is not compatible with zero for all hadron types except for the $\cos 2 \phi$ kaons. The different behavior of kaons with respect to pions suggests a significant contribution to the modulations from scattering off strange quarks, or, more generally, from scattering off the sea, or from fragmentation of light quarks into kaons. 


\section{ACKNOWLEDGMENTS}

We gratefully acknowledge the DESY management for its support and the staff at DESY and the collaborating institutions for their significant effort. This work was supported by the Ministry of Economy and the Ministry of Education and Science of Armenia; the FWO-Flanders and IWT, Belgium; the Natural Sciences and Engineering Research Council of Canada; the National Natural Science Foundation of China; the Alexander von Humboldt Stiftung, the German Bundesministerium für Bildung und Forschung (BMBF), and the Deutsche Forschungsgemeinschaft (DFG); the Italian Istituto Nazionale di Fisica Nucleare (INFN); the MEXT, JSPS, and G-COE of Japan; the Dutch Foundation for Fundamenteel Onderzoek der Materie (FOM); the Russian Academy of Science and the Russian Federal Agency for Science and Innovations; the U.K. Engineering and Physical Sciences Research Council, the Science and Technology Facilities Council, and the Scottish Universities Physics Alliance; the U.S. Department of Energy (DOE) and the National Science Foundation (NSF); the Basque Foundation for Science (IKERBASQUE) and the UPV/EHU under program UFI 11/55; and the European Community Research Infrastructure Integrating Activity under the FP7 "Study of strongly interacting matter (HadronPhysics2, Grant Agreement number 227431)".

\section{Appendix A: Analysis methods}

In this section some technical details particular to this analysis are discussed. Section A 1 includes the details of the fully differential unfolding and fitting procedure. Section A 2 describes the extraction of a $4 D$ model of the measured cosine modulations from the data.

\section{Five-dimensional unfolding and fitting}

As described in section III B, the measured yields are simultaneously unfolded (i.e., corrected for acceptance, smearing and QED radiative effects) and fit by minimizing the $\chi^{2}$ in Eq. 10. In Equation 10, $\sigma^{\text {data }}$ is a vector and $S$ and $C$ are square matrices, all of dimension of the number of bins $(5 * 5 * 6 * 6 * 12=10800$, see Table II). The results vector $\beta$ contains the three fit parameters $(\mathcal{A}, \mathcal{B}$, and $\mathcal{C}$, see Eq. 9$)$ for each $\left(x, y, z, P_{h \perp}\right)$ bin and therefore is of dimension $(5 * 5 * 6 * 6) * 3=2700$. The product $X \beta$ gives the fit function of Eq. 9 in each of the 10800 bins and so $X$ is 10800 by 2700 . Each row contains elements equal to $1, \cos \langle\phi\rangle$, and $\cos 2\langle\phi\rangle$ for that bin; the remaining elements are 0 . The result is that $X$ is block diagonal, with blocks of dimension $12 \times 3$.

The covariance $C$ includes the sum of three sources of statistical uncertainties: the precision of the measured yields, the precision of the Monte Carlo used for the background subtraction and the precision of the Monte Carlo used to construct the smearing matrix ${ }^{3}$. These three sources are accounted for in the covariance following the standard uncertainty propagation. The first two terms are the diagonal covariances of $\sigma_{U U}^{\text {raw }}$ (the raw yields), and $\sigma_{U U}^{\text {backgr }}$ (the background yields). Together, these give the uncertainty of background-subtracted yields, i.e., of $\sigma_{U U}^{\text {data }}=\sigma_{U U}^{\text {raw }}-\sigma_{U U}^{\text {backgr }}$. The third term accounts for the propagation of the smearing matrix covariance $C_{S}$ through the full unfolding and fitting procedure. $C_{S}$ is calculated from the statistical uncertainty of the migration matrix and the Born-level simulated yields used to calculate the smearing matrix. This uncertainty contributes an additive term in $C$ of the form

$$
C^{\text {smear }}=\sigma_{U U}^{\mathrm{unf}} C_{S} \sigma_{U U}^{\mathrm{unf}}{ }^{T},
$$

where $\sigma_{U U}^{\text {unf }}$ is the unfolded (Born-level) yield vector, which is calculated by correcting the measured yields for smearing and background. This third contribution to the covariance is small, as the Monte Carlo productions contain approximately 20 times as many events as the data productions.

The $\chi^{2}$ defined in Eq. 10 was minimized with respect to the vector of parameters $\beta$ by means of linear regression, producing the parameters

$$
\beta=\left(X^{T} S^{T} C^{-1} S X\right)^{-1} X^{T} S^{T} C^{-1} \sigma_{U U}^{\text {data }},
$$

along with their covariance

$$
C_{\beta}=\left(X^{T} S^{T} C^{-1} S X\right)^{-1} .
$$

Here, a generalized procedure was used that unfolds separately each data set with its own smearing matrix, and then simultaneously fits the unfolded yields from the various years ${ }^{4}$. For this purpose, a super matrix form of Eq. 10 was defined,

$$
\chi^{2}=\left(\boldsymbol{\sigma}_{\mathrm{UU}}^{\mathbf{S}}-\mathbf{S}^{\mathbf{S}} X \beta\right)^{T} \mathbf{C}_{\mathbf{S}}^{-\mathbf{1}}\left(\boldsymbol{\sigma}_{\mathrm{UU}}^{\mathbf{S}}-\mathbf{S}^{\mathbf{S}} X \beta\right),
$$

where $\boldsymbol{\sigma}_{\mathbf{U U}}^{\mathrm{S}}$ is a super vector that includes the $\sigma_{U U}^{\text {data, } d}$ for all data sets $d=1, . ., n$

$$
\boldsymbol{\sigma}_{\mathbf{U} U}^{\mathbf{S}}=\left(\begin{array}{c}
\sigma_{U U}^{\text {data }, 1} \\
. . \\
\sigma_{U U}^{\text {data }, n}
\end{array}\right) .
$$

The super matrices $\mathbf{S}^{\mathbf{S}}$ and $\mathbf{C}_{\mathbf{S}}$ include respectively the smearing matrices and their covariances for various data

\footnotetext{
${ }^{3}$ It can be shown mathematically that if the results are first unfolded (including only the first two contributions), and then fit (including the additional uncertainty due to S), an identical term will appear in the calculation of the covariance of the fit parameters.

${ }^{4}$ Mathematically this is equivalent to taking the weighted average of the fit parameters from various data sets before calculating the moments (which correspond to the ratio of the fit parameters).
} 
sets:

$$
\mathbf{S}^{\mathbf{S}}=\left(\begin{array}{c}
S_{1} \\
. . \\
S_{n}
\end{array}\right), \quad \mathbf{C}_{\mathbf{S}}=\left(\begin{array}{ccc}
C_{1}^{-1} & 0 & 0 \\
0 & . . & 0 \\
0 & 0 & C_{n}^{-1}
\end{array}\right) .
$$

Since $\mathbf{C}_{\mathbf{S}}$ is a block-diagonal matrix, equation A4 gives

$$
\begin{gathered}
\beta=\left\{X^{T}\left(\sum_{d=1}^{n} S_{d}^{T} C_{d}^{-1} S_{d}\right) X\right\}^{-1} \\
X^{T}\left(\sum_{d=1}^{n} S_{d}^{T} C_{d}^{-1} \sigma_{U U}^{\mathrm{data}, d}\right)
\end{gathered}
$$

and covariance

$$
C_{\beta}=\left\{X^{T}\left(\sum_{d=1}^{n} S_{d}^{T} C_{d}^{-1} S_{d}\right) X\right\}^{-1} .
$$

\section{2. $4 D$ model of the extracted moments}

Several of the systematic tests described in section IV require a Monte Carlo production that includes azimuthal modulations consistent with those found in the data. To facilitate this, the fully differential final results are fit to a four-dimensional function, which is then used to alter the underlying distribution in an azimuthally independent Pythia6 Monte Carlo production. The fit function used has 38 parameters, 19 for each modulation $(\cos 2 \phi, \cos \phi)$, and is of the form:

$$
\begin{aligned}
f= & A_{1}+A_{2} x+A_{3} y+A_{4} z+A_{5} P_{h \perp} \\
& +A_{6} x^{2}+A_{7} z^{2}+A_{8} P_{h \perp}{ }^{2}+A_{9} x z \\
& +A_{10} x P_{h \perp}+A_{11} z P_{h \perp}+A_{12} y P_{h \perp} \\
& +A_{13} y x+A_{14} y z+A_{15} y^{2} \\
& +A_{16} x^{3}+A_{17} z^{3}+A_{18} P_{h \perp}{ }^{3}+A_{19} y^{3} .
\end{aligned}
$$

Several other functional forms were also tested and gave compatible results. This procedure was used to extract one model separately for hydrogen and deuterium targets, and for each particle type and charge: pions, kaons, and unidentified hadrons.

\section{Appendix B: Hadron Identification}

The Hermes dual-radiator ring-imaging Cherenkov (RICH) detector is described in detail in Ref. [50]. In that article the indirect ray tracing (IRT) particle identification algorithm is presented. In addition, an alternative method, the direct ray tracing (DRT) algorithm was developed, which is described in Ref. [85]. The IRT algorithm calculates an expected Cherenkov angle and compares this to the observed photons. The DRT algorithm performs a simulation, generating an expected photon pattern for a given particle hypothesis, which is then compared to the observed pattern. Because DRT performs a full simulation it is more accurate than IRT, at the cost of increased computing time.

Here, a new method, EVT, is presented. This eventlevel algorithm can more effectively identify tracks in semi-inclusive events where rings from several tracks may overlap.

\section{The EVT algorithm}

The DRT algorithm generates a simulated photomultiplier tube (PMT) hit pattern for each radiator, based on known track kinematics and a particle-type hypothesis. Hit patterns are generated for each particle type hypothesis (pion, kaon, proton) and the likelihood of each hypothesis is calculated by comparing the simulated hit pattern to the hit distribution observed. Due to computing constraints, DRT was initially only used on a subset of particle tracks, and the event-level sum over tracks shown in the equations of section 3.1 of Ref. [85] was not computed. Advances in CPU power in recent years made it possible for the DRT method to be run on all tracks in all events, but the event-level consideration of all tracks in an event was not implemented in the software.

The EVT method implements the full event-level identification algorithm described in Ref. [85]. Simulated hit patterns for each track are combined for all permutations of particle hypotheses to form a set of predicted hit patterns for the event. The likelihood $\left(L^{H}\right)$ of an event hypothesis $H$ is given by the observed hit pattern $\left(C_{\mathrm{PMT}}(i)\right)$ and the probability of hit $\left(P_{\mathrm{PMT}}^{(H)}(i)\right)$,

$$
\begin{aligned}
L^{H}=\sum_{i} \log \left[P_{\mathrm{PMT}}^{H}(i) C_{\mathrm{PMT}}(i)\right. & \\
& \left.+\bar{P}_{\mathrm{PMT}}^{H}(i)\left(1-C_{\mathrm{PMT}}(i)\right)\right],
\end{aligned}
$$

where the probability of no hit is simply $\bar{P}_{\mathrm{PMT}}^{H}(i)=1-$ $P_{\mathrm{PMT}}^{H}(i)$. The observed hit pattern $C_{\mathrm{PMT}}(i)$ is 1 if $\mathrm{PMT}$ $i$ is hit, and 0 otherwise. An event hypothesis $H$ is a set of particle type hypotheses, one for each track in the event. Given $T$ tracks, each with $h(=3$, pions, kaons, proton) possible hypotheses, there are a total of $h^{T}$ event hypotheses $H$. The hypothesis for track $t$ given the event hypothesis $H$ is $H_{t}$. The simulated hit pattern of track $t$, of particle type $H_{t}$, produced from radiator $r$ is given by $N^{\left(H_{t}, t, r\right)}(i)$. The probability $P_{\mathrm{PMT}}^{H}(i)$ of a hit given the event hypothesis $H$, is

$$
\begin{aligned}
& P_{\mathrm{PMT}}^{H}(i)= \\
& \quad 1-\exp \left(-\sum_{r, t}\left[\frac{N^{\left(H_{t}, t, r\right)}(i)}{\sum_{i} N^{\left(H_{t}, t, r\right)}(i)} n^{\left(H_{t}, t, r\right)}\right]-B(i)\right),
\end{aligned}
$$

where the sum is over the hits from both radiators $(r)$ and all the tracks $(t)$ in the event. An unphys- 
ically high number of hits is simulated for each radiator $\left(\sum_{i} N^{\left(H_{t}, t, r\right)}(i)=360\right)$ to construct a smooth distribution of the expected hits. The simulated hit $\operatorname{pattern}\left(N^{\left(H_{t}, t, r\right)}(i)\right)$ is then normalized $\left(\frac{n^{\left(H_{t}, t, r\right)}}{\sum_{i} N^{\left(H_{t}, t, r\right)}(i)}\right)$ to the number of expected PMT hits for the given particle type, track kinematics, and radiator. The total number of expected hits, $n^{\left(H_{t}, t, r\right)}$, is typically $0-10$ hits. The $B(i)$ term is included to take into account physical and experimental backgrounds; see section B 2 for more details.

After the likelihoods $L^{H}$ are computed, the most likely is chosen and the particle type of each track in the event is given by $H_{t}$.

\section{Backgrounds}

The background term $B(i)$ was investigated by counting the average number of hits in each PMT in the absence of tracks in that detector half. Average background values of 0.005 were observed in the data, with a structure that shows the highest values nearest to the beam, indicating that the origin was not only electronic noise. Event displays showed that these "background" events that have no associated tracks in fact show a ring structure in the RICH. Extracting the same quantity from Monte Carlo simulation showed similar results, confirming that these "background" rings are from phys- ical particles not tracked by the spectrometer. They are caused by high-energy photons from $\pi^{0}$ decays and bremsstrahlung of the scattered beam lepton, producing $e^{+} e^{-}$pairs in front of the RICH.

While the $B(i)$ term can only provide an average treatment of such untracked rings, it is the simplest way to take this physical background into account using the existing algorithm. Monte Carlo tests using $B(i)$ determined from data showed that it led to more efficient particle identification with less contamination compared to the constant value of 0.0001 (as was used in the past).

Extracting $B(i)$ from various subsets of the data showed that $B(i)$ is relatively insensitive to the type of target gas. However, it is affected by the gas density, showing higher values for targets with a higher density. When applied to simulated data, a larger $B(i)$ favors identification (both correctly and incorrectly) as a (anti)proton while a smaller $B(i)$ favors identification (both correctly and incorrectly) as a kaon. In each case there is a trade off between efficiency and contamination. Since the overall flux of pions is largest, it is relatively unaffected by the change while the very small antiproton flux shows relatively large changes when using a different $B(i)$. However, the use of different background files had a negligible affect on the cosine modulations extracted here. $B(i)$ was extracted from the unpolarized data for each data year and used for the RICH algorithm applied to all of the data from that year.
[1] J. D. Bjorken and E. A. Paschos, Phys. Rev. 185, 1975 (1969).

[2] R. P. Feynman, Phys. Rev. Lett. 23, 1415 (1969).

[3] D. de Florian, R. Sassot, M. Stratmann, and W. Vogelsang, Phys. Rev. D80, 034030 (2009).

[4] F. D. Aaron et al. (H1 and ZEUS), JHEP 1001, 109 (2010).

[5] J. Pumplin et al., JHEP 0207, 012 (2002).

[6] P. J. Mulders and R. D. Tangerman, Nucl. Phys. B461, 197 (1996).

[7] J. C. Collins and D. E. Soper, Nucl. Phys. B193, 381 (1981).

[8] D. Boer and P. J. Mulders, Phys. Rev. D57, 5780 (1998).

[9] X. Ji, J.-P. Ma, and F. Yuan, Phys. Lett. B597, 299 (2004).

[10] X. Ji, J.-P. Ma, and F. Yuan, Phys. Rev. D71, 034005 (2005).

[11] J. C. Collins, Foundations of Perturbative QCD (Cambridge University Press, London, 2011).

[12] R. Feynman, Photon-hadron interactions, Advanced book classics (Addison-Wesley, 1998).

[13] F. Ravndal, Physics Letters B43, 301 (1973).

[14] R. L. Kingsley, Phys. Rev. D10, 1580 (1974).

[15] A. Kotzinian, Nucl.Phys. B441, 234 (1995).

[16] R. Tangerman and P. Mulders, Phys.Lett. B352, 129 (1995).

[17] R. N. Cahn, Phys. Lett. B78, 269 (1978).

[18] R. N. Cahn, Phys. Rev. D40, 3107 (1989).

[19] D. W. Sivers, Phys. Rev. D41, 83 (1990).
[20] D. W. Sivers, Phys. Rev. D43, 261 (1991).

[21] J. C. Collins, Nucl. Phys. B396, 161 (1993).

[22] S. J. Brodsky, D. S. Hwang, and I. Schmidt, Phys. Lett. B530, 99 (2002).

[23] J. C. Collins, Phys. Lett. B536, 43 (2002).

[24] A. V. Belitsky, X. Ji, and F. Yuan, Nucl. Phys. B656, 165 (2003).

[25] A. Airapetian et al. (HERMES Collaboration), Phys. Lett. B682, 351 (2010).

[26] T. P. Cheng and A. Zee, Phys. Rev. D 6, 885 (1972).

[27] A. Bacchetta et al., JHEP 0702, 093 (2007).

[28] A. Bacchetta, U. D'Alesio, M. Diehl, and C. A. Miller, Phys. Rev. D70, 117504 (2004).

[29] H. Georgi and H. D. Politzer, Phys. Rev. Lett. 40, 3 (1978).

[30] A. Méndez, Nucl. Phys. B145, 199 (1978).

[31] M. Glück, E. Reya, and A. Vogt, Eur. Phys. J. C5, 461 (1998).

[32] D. de Florian, R. Sassot, and M. Stratmann, Phys. Rev. D75, 114010 (2007).

[33] S. Wandzura and F. Wilczek, Phys. Lett. B72, 195 (1977).

[34] J. J. Aubert et al. (European Muon Collaboration), Phys. Lett. B130, 118 (1983).

[35] M. Arneodo et al. (European Muon Collaboration), Z. Phys. C34, 277 (1987).

[36] J. Breitweg et al. (Zeus Collaboration), Phys. Lett. B481, 199 (2000).

[37] M. R. Adams et al. (E665 Collaboration), Phys. Rev. 
D48, 5057 (1993).

[38] M. Osipenko et al. (ClAs Collaboration), Phys. Rev. D80, 032004 (2009).

[39] G. Sbrizzai (Compass Collaboration), J. Phys.: Conf. Ser. 295, 012043 (2011).

[40] S. Falciano et al. (NA10 Collaboration), Z. Phys. C31, 513 (1986).

[41] M. Guanziroli et al. (NA10 Collaboration), Z. Phys. C37, 545 (1988).

[42] J. S. Conway et al., Phys. Rev. D39, 92 (1989).

[43] J. G. Heinrich et al., Phys. Rev. D44, 1909 (1991).

[44] L. Y. Zhu et al. (FNAL-E866/NuSea Collaboration), Phys. Rev. Lett. 99, 082301 (2007).

[45] L. Y. Zhu et al. (FNAL E866/NuSea Collaboration), Phys. Rev. Lett. 102, 182001 (2009).

[46] C. S. Lam and W.-K. Tung, Phys. Rev. D21, 2712 (1980).

[47] D. Boer and P. J. Mulders, Nucl. Phys. B569, 505 (2000).

[48] K. Ackerstaff et al. (Hermes Collaboration), Nucl. Instrum. Meth. A417, 230 (1998).

[49] R. Frühwirth, Nucl. Instrum. Meth. A262, 444 (1987).

[50] N. Akopov et al., Nucl. Instrum. Meth. A479, 511 (2002).

[51] H. E. Jackson (Hermes Collaboration), Nucl. Instrum. Meth. A553, 205 (2005).

[52] H. Avakian et al., Nucl. Instrum. Meth. A417, 69 (1998).

[53] T. Sjöstrand et al., Comput. Phys. Commun. 135, 238 (2001).

[54] T. Sjöstrand, Comput. Phys. Commun. 82, 74 (1994).

[55] A. Airapetian et al. (Hermes Collaboration), JHEP 1008, 130 (2010).

[56] I. Akushevich, H. Böttcher, and D. Ryckbosch (1998), hep-ph/9906408.

[57] R. Brun, R. Hagelberg, M. Hansroul, and J. Lassalle, CERn Report CERn-DD-78-2-REV (1978).

[58] G. Ingelman, A. Edin, and J. Rathsman, Comput. Phys. Commun. 101, 108 (1997).

[59] F. Giordano (2008), proceedings of Second International Workshop on Transverse Polarisation Phenomena in Hard Processes (Transversity 2008), Ferrara, Italy, 28 - 31 May 2008, World Scientific, 2008, p. 177.

[60] Durham HEP database, http://durpdg.dur.ac.uk; INSPIRE, http://inspirebeta.net/record/1111237/; mail-to: management@hermes.desy.de.

[61] http://www-hermes.desy.de/cosnphi/.

[62] A. Airapetian et al. (Hermes Collaboration), Multiplicities of charged pions and kaons from semi-inclusive deep- inelastic scattering on the proton and the deuteron (in preparation).

[63] D. de Florian, R. Sassot, and M. Stratmann, Phys. Rev. D76, 074033 (2007).

[64] G. Schnell (HeRmes Collaboration) (2007), talk delivered at 'Transverse momentum, spin, and position distributions of partons in hadrons' ECT* $\left.^{*} 2007\right)$, Trento, Italy, 11 - 15 Jun 2007.

[65] A. Airapetian et al. (Hermes Collaboration), Phys. Rev. Lett. 94, 012002 (2005).

[66] M. Anselmino et al., Phys. Rev. D75, 054032 (2007).

[67] A. Airapetian et al. (Hermes Collaboration), Phys. Lett. B693, 11 (2010).

[68] V. Y. Alexakhin et al. (Compass Collaboration), Phys. Rev. Lett. 94, 202002 (2005).

[69] K. Abe et al. (Belle Collaboration), Phys. Rev. Lett. 96, 232002 (2006).

[70] R. Lamb, Ph.D. thesis, University of Illinois at UrbanaChampaign (2010), DESY-THESIS-2010-035.

[71] V. Barone, S. Melis, and A. Prokudin, Phys. Rev. D81, 114026 (2010).

[72] M. Burkardt and B. Hannafious, Phys. Lett. B658, 130 (2008).

[73] M. Burkardt, Phys. Rev. D72, 094020 (2005).

[74] L. P. Gamberg, G. R. Goldstein, and K. A. Oganessyan, Phys. Rev. D67, 071504 (2003).

[75] L. P. Gamberg, G. R. Goldstein, and M. Schlegel, Phys. Rev. D77, 094016 (2008).

[76] V. Barone, A. Prokudin, and B.-Q. Ma, Phys. Rev. D78, 045022 (2008).

[77] B. Zhang, Z. Lu, B.-Q. Ma, and I. Schmidt, Phys. Rev. D78, 034035 (2008).

[78] F. Giordano and R. Lamb (Hermes Collaboration), AIP Conf. Proc. 1149, 423 (2009).

[79] M. Anselmino et al., Phys. Rev. D72, 094007 (2005).

[80] M. Anselmino et al., Phys. Rev. D71, 074006 (2005).

[81] S. M. Aybat and T. C. Rogers, Phys. Rev. D83, 114042 (2011).

[82] M. Anselmino, M. Boglione, A. Prokudin, and C. Turk, Eur. Phys. J. A31, 373 (2007).

[83] X. Artru, J. Czyzewski, and H. Yabuki, Z. Phys. C73, 527 (1997).

[84] A. Airapetian et al. (Hermes Collaboration), Phys. Lett. B666, 446 (2008).

[85] E. Cisbani, Nuclear Physics B - Proceedings Supplements 78, 366 (1999). 